(C) 1998 Springer-Verlag New York Inc.

\title{
A Finite-Dimensional Dynamical Model for Gelation in Coagulation Processes
}

\author{
F. P. da Costa \\ Instituto Superior Técnico, Departamento de Matemática, Avenida Rovisco Pais, 1, P-1096 \\ Lisboa, Portugal \\ e-mail: fcosta@math.ist.utl.pt
}

Received April 26, 1997; revised October 3, 1997; accepted October 23, 1997

Communicated by Philip Holmes

Summary. We study a finite-dimensional system of ordinary differential equations derived from Smoluchowski's coagulation equations and whose solutions mimic the behaviour of the nondensity-conserving (geling) solutions in those equations.

The analytic and numerical studies of the finite-dimensional system reveals an interesting dynamic behaviour in several respects: Firstly, it suggests that some special geling solutions to Smoluchowski's equations discovered by Leyvraz can have an important dynamic role in gelation studies, and, secondly, the dynamics is interesting in its own right with an attractor possessing an unexpected structure of equilibria and connecting orbits.

\section{Introduction}

The kinetics of cluster growth can be modelled by the following infinite system of ordinary differential equations, first proposed by Smoluchowski in his study of coagulation of colloid particles animated by Brownian motion [30],

$$
\dot{c}_{j}=\frac{1}{2} \sum_{k=1}^{j-1} a_{j-k, k} c_{j-k} c_{k}-c_{j} \sum_{k=1}^{\infty} a_{j, k} c_{k}, \quad j=1,2,3, \ldots,
$$

where $c_{j}=c_{j}(t) \geq 0$ denotes the concentration of clusters made up of $j$ identical particles ( $j$-clusters, for short), the time-independent parameters $a_{j, k}=a_{k, j} \geq 0$ are the rate coefficients for the coagulation reactions

$$
(j \text {-cluster })+(k \text {-cluster }) \longrightarrow((j+k) \text {-cluster }),
$$

and equations (1) are written assuming the validity of the mass action law of chemical kinetics (the first sum in the right-hand side of (1) is defined to be zero if $j=1$ ). 
These equations, as well as generalizations allowing for the fragmentation of clusters, and their continuous cluster-sizes analogues, have been used in a variety of applications, including astrophysics [9], [26], aerosol and colloidal sciences [14], [15], and chemistry and chemical engineering [1], [5], [29], [32]. It is believed that the relevant physical details of the situation under study determine the form of the rate coefficients $a_{j, k}$. In order to gain some understanding of why this is so, consider the following simple argument. Let the mass of a single particle be equal to 1 . Then, the mass of a $j$-cluster is equal to $j$. Assuming the clusters are incompressible, the volume of a $j$-cluster will be proportional to $j$; if the clusters are spherical, the surface area will grow like $j^{2 / 3}$, and its diameter like $j^{1 / 3}$. Thus, a coagulation mechanism dependent on the surface area available for reaction will lead naturally to coefficients $a_{j, k}$ containing the power $2 / 3$ of $j$ and $k$. If the mechanism is essentially dependent on the linear dimensions of the clusters, the power $1 / 3$ will show up. Likewise, the power 1 will be connected with mechanisms essentially dependent on the total mass (or volume) of the clusters. Clearly, other powers are possible if the coagulation mechanism is not dependent on a single factor, or if the clusters are not approximately spherical, or if they have preferential "active" sites, etc. The case of linear-chain polymerization is a good example: In this situation the active sites for the reactions are the end-points of the linear polymeric molecules, and there are only two of them, independent of the mass (=volume=length) of the chain. Hence, one expects the coefficients $a_{j, k}$ to be essentially independent of $j$ and $k$. The particulars of the interaction mechanism are reflected in the specific form of the kernel (not only on the powers involved), of which many different types have been proposed. In Table 1, adapted from [5], [12], [25], [29], we present some of the coagulation kernels most common in the scientific literature.

Due to the complicated, and usually quite special, forms of most of the rate coefficients, the large majority of the rigorous mathematical results about solutions to these equations have been obtained using some sort of idealized form for the coagulation kernel, which is sufficiently simple to allow a rigorous analysis, but, nevertheless, is still believed to capture some, although probably not much, of the real particularities of the phenomenon being modelled. In this context, the simplest classes of rate coefficients are the sum type $a_{j, k}=r_{j}+r_{k}$, and the product type $a_{j, k}=r_{j} r_{k}$, where in both cases $\left(r_{j}\right)$ is a nonnegative sequence.

Among the various questions that have been asked about equations (1) and their solutions, a particularly important one is related to the possibility of using them as a model for the sol-gel phase transition. In order to describe the problem, remember the assumption that a $j$-particle cluster has mass $j$. Then, the quantity $j c_{j}(t)$ is the concentration of $j$-clusters at time $t$, and the total density of the system described by (1) is given by $\rho(t):=\sum_{j=1}^{\infty} j c_{j}(t)$. Since each elementary reaction (2) conserves mass, it is expected that $\rho(t)$ is independent of $t$. This is indeed what happens at a formal level: Assuming $\rho(t)$ to be differentiable and such that $\dot{\rho}(t)=\sum_{j=1}^{\infty} j \dot{c}_{j}(t)$, substituting $\dot{c}_{j}(t)$ given by (1) in the series and computing formally, the result is $\dot{\rho}(t)=0$, for all $t$. However, it is known that the formal computation just suggested cannot be made rigorous in all situations: For some rate coefficients $a_{j, k}$ increasing sufficiently fast with $j$ and $k$, it has been proved rigorously that $\rho(t)$ cannot be constant for all $t$, and that there must exist a nonnegative time $t_{g}$ such that $\rho(t)=\rho(0)$ for $t \leq t_{g}$ but $\rho(t)<\rho(0)$ for $t>t_{g}$ [6], [7], [18], [20], [24], [27], [28]. In the literature this phenomenon is called 
Table 1. Some commonly used coagulation kernels $a_{j, k}$.

\begin{tabular}{ll}
\hline \multicolumn{1}{c}{$a_{j, k}$} & \multicolumn{1}{c}{ Comment } \\
\hline 1 & $\begin{array}{l}\text { Approximate Brownian coagulation, } \\
\text { Linear-chain polymerization. }\end{array}$ \\
& $\begin{array}{l}\text { Branched-chain polymerization of } A R B_{f-1} \text { type (large } f \text { limit), } \\
\text { Limit case of gravitation coagulation. }\end{array}$ \\
$j^{-2 / 3}+k^{-2 / 3}$ & Diffusion-controlled growth of supported metal crystallites. \\
$j^{k}$ & Branched-chain polymerization of $R A_{f}$ type (large $f$ limit). \\
$\left(j^{1 / 3}+k^{1 / 3}\right)\left(j^{-1 / 3}+k^{-1 / 3}\right)$ & Brownian coagulation (continuum regime). \\
$\left(j^{1 / 3}+k^{1 / 3}\right)^{2}\left(j^{-1}+k^{-1}\right)^{1 / 2}$ & Brownian coagulation (free molecular regime). \\
$\left(j^{1 / 3}+k^{1 / 3}\right)^{3}$ & Shear (linear velocity profile). \\
$\left(j^{1 / 3}+k^{1 / 3}\right)^{7}$ & Shear (nonlinear velocity profile). \\
$\left(j^{1 / 3}+k^{1 / 3}\right)^{2}\left|j^{1 / 3}-k^{1 / 3}\right|$ & Gravitational settling (particles larger than $\sim 50 \mu m)$. \\
\hline
\end{tabular}

gelation, and it is interpreted physically as corresponding to the occurrence of a dynamic phase transition in the system. Both equations (1) and density $\rho(t)$ take into account only "microscopic" clusters with a finite, although arbitrarily large, number of particles. If the rate of production of clusters is very high, a portion of the total mass of the system is rapidly transferred to larger and larger clusters, and can be carried to a "macroscopic" cluster with an infinite number of particles (i.e., $j \rightarrow \infty$ ) in a finite time $t_{g}$. This new phase, called the gel, is not modelled by equations (1) and has a mass proportional to $\rho(0)-\rho(t)$ for $t>t_{g}$.

This dynamic phase transition has attracted a good deal of interest in recent years, and a large body of literature has been built in trying to characterize the rate coefficients and initial conditions for which gelation occurs, as well as the properties of the geling solutions (see, for instance, [5], [13], [16], [18], [20], [27], [29], [31], [33].) However, surprisingly little is known on a mathematically rigorous basis, even for relatively simple rate coefficients.

The possibility of occurrence of gelation for sum type coefficients has been settled negatively; in particular, the following has been established rigorously:

(S1) If $r_{j} \leq A j$, for some positive constant $A$, then all solutions conserve density $\rho(t)$ for all $t[3]$.

(S2) If $r_{j} \geq A j^{\alpha}$, where $A>0$ and $\alpha>1$ are constants, then system (1) has no nonzero solutions, in any time interval [0,T], for $T>0$ [3], [7].

This means that no gelation can occur in these cases. 
For product-type kernels, the following has been proved:

(P1) If $r_{j} \leq A j^{\alpha}$, with $A \geq 0$ and $\alpha \leq 1 / 2$ constants, then all solutions conserve density $\rho(t)$ for all $t[3]$.

(P2) If $r_{j} \geq A j^{\alpha}$, with $A \geq 0$ and $\alpha>1$ constants, then all solutions exhibit gelation with $t_{g}=0$ [7].

(P3) If $r_{j}=A j+B$, where $A$ and $B$ are nonnegative constants, then all solutions exhibit gelation at an instant $t_{g} \in[0, \infty)$ and if the initial condition satisfies $\sum_{j=1}^{\infty} j^{2} c_{j}(0)<\infty$, then $t_{g}>0$ [27].

(P4) When $r_{j}=A j$, the following explicit solution was obtained by Leyvraz and Tschudi in [20] (see also [2], [28]) for monodispersed monomeric initial data (i.e., $c_{1}(0)=1$, and $c_{j}(0)=0, j \geq 2$, or, more conveniently, $c_{j}(0)=\delta_{j, 1}$, where $\delta_{j, i}$ is the Kronecker symbol):

$$
c_{j}(t)= \begin{cases}\frac{j^{j-2}}{j !}\left(A^{2} t\right)^{j-1} e^{-A^{2} j t}, & t \leq A^{-2}, \\ \frac{j^{j-2} e^{-j}}{j !} \frac{1}{A^{2} t}, & t>A^{-2} .\end{cases}
$$

The density $\rho(t)$ of this solution is

$$
\rho(t)= \begin{cases}1, & t \leq A^{-2}, \\ \frac{1}{A^{2} t}, & t>A^{-2},\end{cases}
$$

and so a precise gelation time $t_{g}=A^{-2}$ and post-gel asymptotic behaviour of the solutions were obtained. We will refer to (3) as the Leyvraz-Tschudi solution.

(P5) For $r_{j} \geq A j^{\alpha}$, with $A>0$ and $\alpha>1 / 2$ constants, and $r_{j}>r_{1}$ for all $j \geq 2$, there exists a positive sequence $\left(\alpha_{j}\right)$ such that $\sum_{j=1}^{\infty} j \alpha_{j}<\infty$ and $c_{j}(t)=\alpha_{j}(t+C)^{-1}$ is a solution to (1) for all $t \geq 0$ and constant $C>0$. This result was proved by Leyvraz in [18] and will play an important role in the present paper. We will call them the Leyvraz solutions and the sequence $\left(\alpha_{j}\right)$ will be referred to as the Leyvraz sequence.

The results quoted above show that, for product-type coefficients, the behaviour of solutions is known when $r_{j}$ has asymptotic growth like $j^{\alpha}$, for both $\alpha \leq 1 / 2$ and $\alpha>1$. The intermediate case $1 / 2<\alpha \leq 1$ is poorly understood, and the conjecture that also in this case all nonzero solutions exhibit gelation [16], [21], [33] remains one of the main open problems in this area.

In the present study we do not either prove the conjecture to hold or disprove it, but instead we report on a finite-dimensional system obtained from a truncation of the infinite system (1) whose solutions, in some sense, mimic the behaviour of the geling solutions of (1) refered to in (P4) and (P5) above. The analysis of the finite-dimensional system allows us to identify an infinite family of Leyvraz-type geling solutions to (1), of which the Leyvraz solution in [18] is but one element. The analytical and numerical studies of the finite-dimensional system show a dynamical behaviour that is very interesting in its own right, even if this approach will turn out to be useless for the gelation problem. More specifically, these studies seem to indicate that the attractor of the finite-dimensional 
system has an unexpected structure of equilibria and connecting orbits. The equilibria are related closely to the Leyvraz-type geling solutions referred to above, and the numerics seem to point to a possibly important dynamic role played by the Leyvraz-type solutions in the infinite-dimensional case.

The paper is organized as follows: In Section 2 we derive the finite-dimensional system and study its basic properties. In Section 3 we study the equilibria of the finitedimensional system and use the result to prove the existence of an infinite family of geling solutions to the Smoluchowski equations (1). In Section 4 we study the dynamics of the finite-dimensional system. Finally, in Section 5 we briefly discuss the possible relevance of our study to the elucidation of the gelation behaviour in the original system (1).

\section{Derivation of the Finite-Dimensional System and Basic Properties of Solutions}

Most of the mathematical studies of Smoluchowski's coagulation equations or of more general analogues, such as the Becker-Döring and the Coagulation-Fragmentation equations, use at some point the technique of approximating the infinite system of equations by a finite $n$-dimensional truncation and proving results for the infinite system by passing to the limit $n \rightarrow \infty$ in the solutions to the $n$-dimensional truncations (see, for example, [3], [4], [8], [24]).

It is important for the present paper to state exactly what is meant by a finitedimensional truncation of the Smoluchowski equations (1), and this will be done next.

\subsection{Finite-Dimensional Truncations}

By a finite $n$-dimensional truncation of (1), we mean a system of $n$ ordinary differential equations, in the phase variables $c_{j}$ for $j=1,2, \ldots, n$, obtained from (1) by making $a_{j, k}=0$ outside of a finite subset of $\mathbb{N} \times \mathbb{N}$. Since, for each $j$, the first sum in the righthand side of (1) has only terms $c_{k}$ with $k<j$, it is reasonable to consider truncations that preserve this sum, so a finite $n$-dimensional truncation must satisfy the following

(i) $c_{1}, \ldots, c_{n}$ are the only phase variables $\left(c_{n+1}, c_{n+2}, \ldots \equiv 0\right)$.

(ii) The first sum in the right-hand side of (1) must remain unchanged, for $j=1, \ldots, n$.

These two constraints, together with the basic symmetry condition $a_{j, k}=a_{k, j}$, allow us to classify the truncations using the sets of pairs of indices $(j, k)$ for which the rate coefficients $a_{j, k}$ remain unchanged by the truncation. More precisely, each $n$-dimensional truncation of (1) corresponds to a subset $U^{n}$ of $\mathbb{N} \times \mathbb{N}$ which is symmetric with respect to reflection on the line $k=j$, contained in the square $[1, n]^{2}$, and containing the triangle $\{(j, k) \in \mathbb{N} \times \mathbb{N}: j+k \leq n\}$. The $U^{n}$-truncated system is obtained from (1) simply by setting $a_{j, k}=0$ if $(j, k) \notin U^{n}$. Of all the $n$-dimensional truncations, the two extremal ones are particularly important:

(a) The maximal $n$-truncation, corresponding to the set

$$
U_{\max }^{n}=\{(j, k) \in \mathbb{N} \times \mathbb{N}: j+k \leq n\},
$$


which sets to zero the maximum possible number of rate coefficients $a_{j, k}$, is the system

$$
\dot{c}_{j}=\frac{1}{2} \sum_{k=1}^{j-1} a_{j-k, k} c_{j-k} c_{k}-c_{j} \sum_{k=1}^{n-j} a_{j, k} c_{k}, \quad j=1,2, \ldots, n .
$$

(b) The minimal $n$-truncation, corresponding to the set

$$
U_{\min }^{n}=\{(j, k) \in \mathbb{N} \times \mathbb{N}: \max \{j, k\} \leq n\},
$$

which sets to zero the smallest possible number of rate coefficients compatible with the restrictions above, is the system

$$
\dot{c}_{j}=\frac{1}{2} \sum_{k=1}^{j-1} a_{j-k, k} c_{j-k} c_{k}-c_{j} \sum_{k=1}^{n} a_{j, k} c_{k}, \quad j=1,2, \ldots, n .
$$

Clearly, any other $U^{n}$-truncation will satisfy $U_{\max }^{n} \subset U^{n} \subset U_{\min }^{n}$.

\subsection{The Minimally Truncated Smoluchowski Equations}

Most of the mathematical studies of (1) have made use of the maximal truncation and, since solutions $\left(c^{n}\right)$ to the maximally $n$-truncated system conserve the truncated density $\rho_{n}(t):=\sum_{j=1}^{n} j c_{j}^{n}(t)[3]$, this truncation is well-suited for proving density conservation of solutions to the full system (1). However, being interested in gelation, i.e., in the breakdown of density conservation at a certain $t_{g} \geq 0$, we would like to have a finite $n$-dimensional system for which solutions do not conserve density $\rho_{n}(t)$. In this case the minimal truncation is more adequate; just from an heuristic argument we see that the truncated density is not expected to be conserved by the minimally truncated system: Suppose $a_{j, k}>0$ if and only if $(j, k) \in U_{\min }^{n}$. Take, for example, $j=k=n$. Then, as $(n, n) \in U_{\min }^{n}$ we have $a_{n, n}>0$, which means that the reaction

$$
(n \text {-cluster })+(n \text {-cluster }) \longrightarrow((2 n) \text {-cluster })
$$

occurs with a nonzero velocity, and so some clusters of sizes larger than $n$ are formed. As the truncated density is computed by summing up over all clusters with sizes not larger than $n$, one expects $\rho_{n}(t)$ to decrease with $t$ because part of the mass of the system is transferred to clusters of sizes from $n+1$ to $2 n$ that are not accounted for either in the truncated system or in the truncated density. If we remember the physical interpretation of gelation discussed in the introduction, we can draw a parallel between the formation of a gel phase in (1) and the formation of clusters of sizes larger than $n$ in the minimally $n$-truncated system: Neither is modelled directly by the differential equations, but their presence is felt by the decrease in density of the solutions. That the minimally $n$-truncated system can, in this way, model gelation, or other precipitation phenomena, has already been pointed out in [19], and for a slightly different concept of truncation, in [2], [23]. These last papers (and also [17]) use a $2 n$-dimensional truncation obtained by application of the minimal $n$-truncation described in 2.1(b) to system (1) without the restriction of considering $c_{1}, \ldots, c_{n}$ as the only phase variables of the truncated system, 
that is, dropping the requirement (i) in 2.1. This gives rise to a system of $2 n$ ordinary differential equations for the phase variables $c_{1}, \ldots, c_{2 n}$, of which the equations for $c_{1}, \ldots, c_{n}$ are exactly (5), and the equations for $c_{n+1}, \ldots, c_{2 n}$ are

$$
\dot{c}_{j}=\frac{1}{2} \sum_{k=j-n}^{n} a_{j-k, k} c_{j-k} c_{k}, \quad j=n+1, \ldots, 2 n .
$$

Thus, the minimally truncated system is equivalent to the first $n$ equations of the $2 n$ truncation used in [2], [23].

We now start a mathematical study of the minimally $n$-truncated system (5).

Local existence, uniqueness, regularity, and continuous dependence of solutions to (5) are obtained by standard arguments in the theory of ordinary differential equations. We next prove that the nonnegative cone of $\mathbb{R}^{n}$ is invariant for (5).

Proposition 1. Let $c(t)=\left(c_{j}(t)\right)_{1 \leq j \leq n}$ be the solution of $(5)$ on $[0, T)$, for some $T>0$, with initial data $c(0)=c_{0} \in \mathbb{R}_{0}^{n+}$. Then $c(t) \in \mathbb{R}_{0}^{n+}$, for all $t \in[0, T)$.

Proof. For each $1 \leq j \leq n$, define

$$
\begin{aligned}
R_{j}(t) & :=\frac{1}{2} \sum_{k=1}^{j-1} a_{j-k, k} c_{j-k}(t) c_{k}(t), \\
\varphi_{j}(t) & :=\sum_{k=1}^{n} a_{j, k} c_{k}(t) \\
E_{j}(t) & :=\exp \left(\int_{0}^{t} \varphi_{j}(s) d s\right) .
\end{aligned}
$$

We can write (5) in the following integral form:

$$
c_{j}(t) E_{j}(t)=c_{0 j}+\int_{0}^{t} E_{j}(s) R_{j}(s) d s, \quad 1 \leq j \leq n .
$$

Clearly, $E_{j}(t)>0$ for all $t$ and $j$. Then, for $j=1$ we have $R_{1}(t) \equiv 0$ by definition, and $c_{1}(t) E_{1}(t)=c_{01} \geq 0$. Hence $R_{2}(t)=\frac{1}{2} a_{1,1}\left(c_{1}(t)\right)^{2} \geq 0$ and so, for $j=2$,

$$
c_{2}(t) E_{2}(t)=c_{02}+\int_{0}^{t} E_{2}(s) R_{2}(s) d s \geq c_{02} \geq 0 .
$$

Since $R_{j}(t)$ contains only components $c_{k}(t)$ with $k<j$, we can proceed successively until $j=n$, obtaining the result.

We can obtain a much better characterization of the positivity properties of the solutions to (5) if we consider $a_{j, k}>0$ in (5). In this case we have the following result, which was proved originally in [11, Theorem 2] for the maximally truncated system, but whose proof can be easily modified for the present case: 
Proposition 2. Let $c(t)=\left(c_{j}(t)\right)_{1 \leq j \leq n}$ be the solution of $(5)$ on $[0, T)$, for some $T>0$, with initial data $c(0)=c_{0} \in \mathbb{R}_{0}^{n+}$. Let $\mathcal{J}(t)=\left\{j \in \mathbb{N}: c_{j}(t)>0\right\}$ and $P=\mathcal{J}(0)$. Then, for all $t>0, \mathcal{J}(t) \equiv \mathcal{J}$ is independent of $t$ and is given by

$$
\mathcal{J}=\{1, \ldots, n\} \cap \operatorname{span}_{\mathbb{N}_{0}}(P),
$$

where $\operatorname{span}_{\mathbb{N}_{0}}(P):=\left\{j=\sum_{i} n_{i} p_{i}: p_{i} \in P, n_{i} \in \mathbb{N}_{0}\right.$, and $\left.\max _{i} n_{i}>0\right\}$.

We now prove that the density $\rho_{n}(t)$ is a Lyapunov functional for (5), and that solutions are defined for all positive $t$.

Proposition 3. Let $c(t)=\left(c_{j}(t)\right)_{1 \leq j \leq n}$ be any solution of $(5)$ on $[0, T)$, for some $T>0$. Then, there is a continuation $\hat{c}(t)$ of $c(t)$ to $[0, \infty)$ and $\sum_{j=1}^{n} j \hat{c}_{j}(t)$ is nonincreasing in $[0, \infty)$.

Proof. We start by observing that

$$
\begin{aligned}
\dot{\rho}_{n}(t)= & \frac{1}{2} \sum_{j=2}^{n} \sum_{k=1}^{j-1} j a_{j-k, k} c_{j-k} c_{k}-\sum_{j=1}^{n} \sum_{k=1}^{n} j a_{j, k} c_{j} c_{k} \\
& \text { (changing the order of summation in the first double sum) } \\
= & \frac{1}{2} \sum_{k=1}^{n-1} \sum_{j=k+1}^{n}((j-k)+k) a_{j-k, k} c_{j-k} c_{k}-\sum_{j=1}^{n} \sum_{k=1}^{n} j a_{j, k} c_{j} c_{k} \\
& (\text { changing variables } j \mapsto \ell:=j-k, \text { and using the } \\
& \text { symmetry of the summand in the first double sum) } \\
= & \sum_{k=1}^{n-1} \sum_{\ell=1}^{n-k} k a_{\ell, k} c_{\ell} c_{k}-\sum_{k=1}^{n} \sum_{j=1}^{n} j a_{j, k} c_{j} c_{k} \\
= & -\sum_{j=1}^{n} \sum_{k=n-j+1}^{n} j a_{j, k} c_{j} c_{k} \leq 0 .
\end{aligned}
$$

Then, for all $1 \leq j \leq n$ and $t \in[0, T)$,

$$
0 \leq c_{j}(t) \leq j^{-1} \sum_{j=1}^{n} j c_{j}(0),
$$

and, since the right-hand side of (5) is a polynomial in the $c_{j}$ 's, this implies that $c_{j}(t)$ has a continuation $\hat{c}(t)$ to $[0, \infty)$, and $\sum_{j=1}^{n} j \dot{\hat{c}}_{j}(t) \leq 0$ for all $t \geq 0$.

The inequality (6) can be improved using the positivity result of Proposition 2:

Proposition 4. Let $a_{j, k}>0$ for all $(j, k) \in U_{\min }^{n}$. Assume $c_{0} J \neq 0$ for some $J \in$ $\{1, \ldots, n\}$, and let $c(t)$ be the solution of $(5)$ in $[0, \infty)$ with initial data $c_{0}$. Then $\rho_{n}(t)$ is strictly decreasing for all $t>0$. 
Proof. By Proposition 2, we have $c_{j}(t)>0$ for all positive $t$, and all $j \in\{1, \ldots, n\} \cap$ $(J \cdot \mathbb{N})$, where $J \cdot \mathbb{N}:=\{j=J n: n \in \mathbb{N}\}$. Let $\mu=\lfloor n / J\rfloor:=\max \{m \in \mathbb{N}: m \leq n / J\}$. Thus, $(\mu+1) J>n$, which implies $n-\mu J+1<J+1$, and since $n-\mu J+1$ is an integer, we must have $n-\mu J+1 \leq J$. Hence,

$$
\begin{aligned}
\sum_{j=1}^{n} \sum_{k=n-j+1}^{n} j a_{j, k} c_{j}(t) c_{k}(t) & \geq \mu J c_{\mu J}(t) \sum_{k=n-\mu J+1}^{n} a_{\mu J, k} c_{k}(t) \\
& \geq \mu J a_{\mu J, J} c_{\mu J}(t) c_{J}(t)>0,
\end{aligned}
$$

and so, using (6), $\dot{\rho}_{n}(t)<0$.

We can use this behaviour of the density to obtain the general asymptotic behaviour of nonnegative solutions to (5).

Proposition 5. With the assumptions of Proposition 4, $c(t) \longrightarrow 0$ as $t \rightarrow \infty$.

Proof. Let $c(t)$ be a nonnegative solution of (5). By Proposition 4, the density $\rho_{n}(c(t))$ is a differentiable Lyapunov functional for (5), with minimum equal to 0 , which is attained at $c=0$. As $\mathbb{R}_{0}^{n+}$ is positively invariant for (5), the result follows by standard arguments.

We also can prove easily that all solutions to the infinite system (1) converge componentwise to zero as $t \rightarrow \infty$, provided that at least the diagonal coefficients $a_{j, j}$ are positive for all $j,[8$, Theorem 4.3].

The fact that nonzero solutions to (5) converge to the zero solution as $t \rightarrow \infty$, and have a strictly decreasing density, is in agreement with what happens with the Leyvraz solutions of (1) (see (P5) in the introduction), but this does not necessarily mean that they are a good approximation of the Leyvraz geling solutions, and even less of the geling solutions with positive geling time $t_{g}$ for which density is constant in $\left[0, t_{g}\right]$, such as in the case of the Leyvraz-Tschudi solutions in (P4). An indication that solutions to (5) can, in fact, be a good approximation to geling solutions of (1) is given by numerically solving (5) with parameters and initial conditions for which there exists a known geling solution of (1) and comparing the results. In Figure 1 we plot the density $\rho_{n}(t)$ as a function of $t$ for numerical solutions to (5) with $a_{j, k}=j k$ and initial data $c_{j}(0)=\delta_{j, 1}$, for several values of $n$; together with these plots, we show the density (4) of the Leyvraz-Tschudi solution (3) for the same rate constants (i.e., $A=1$ ). The numerical evidence seems to indicate that the density of solutions to (5) for large $n$ approximate the density of the corresponding exact solutions to (1) rather well.

Similar evidence can be obtained easily for each component $c_{j}$ of the solution, and in this case we can easily prove the following convergence result.

Proposition 6. Assume $a_{j, k} \leq A(j k)^{\alpha}$ with $A>0$ and $\alpha \leq 1$ constants. Let $c_{0}=$ $\left(c_{0 j}\right)_{j \in \mathbb{N}}$ be such that $c_{0 j} \geq 0$ and $\sum_{j=1}^{\infty} j c_{0 j}<\infty$. Let $c^{n}=\left(c_{j}^{n}\right)_{1 \leq j \leq n}$ be the solution to (5) with initial data $c_{j}(0)=c_{0}$ for $1 \leq j \leq n$. Then, there exists a solution $c=\left(c_{j}\right)_{j \in \mathbb{N}}$ of (1) such that, for all $t \geq 0$ and all fixed $j$,

$$
c_{j}^{n}(t) \longrightarrow c_{j}(t), \quad \text { as } n \rightarrow \infty \text {. }
$$




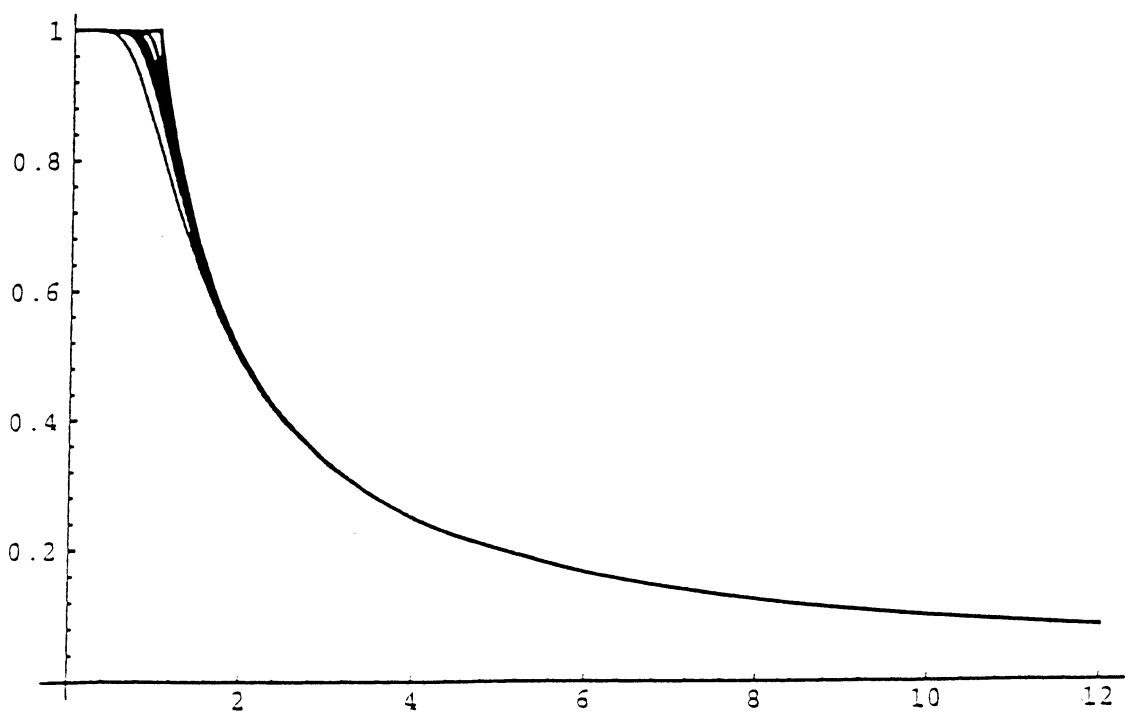

(a)

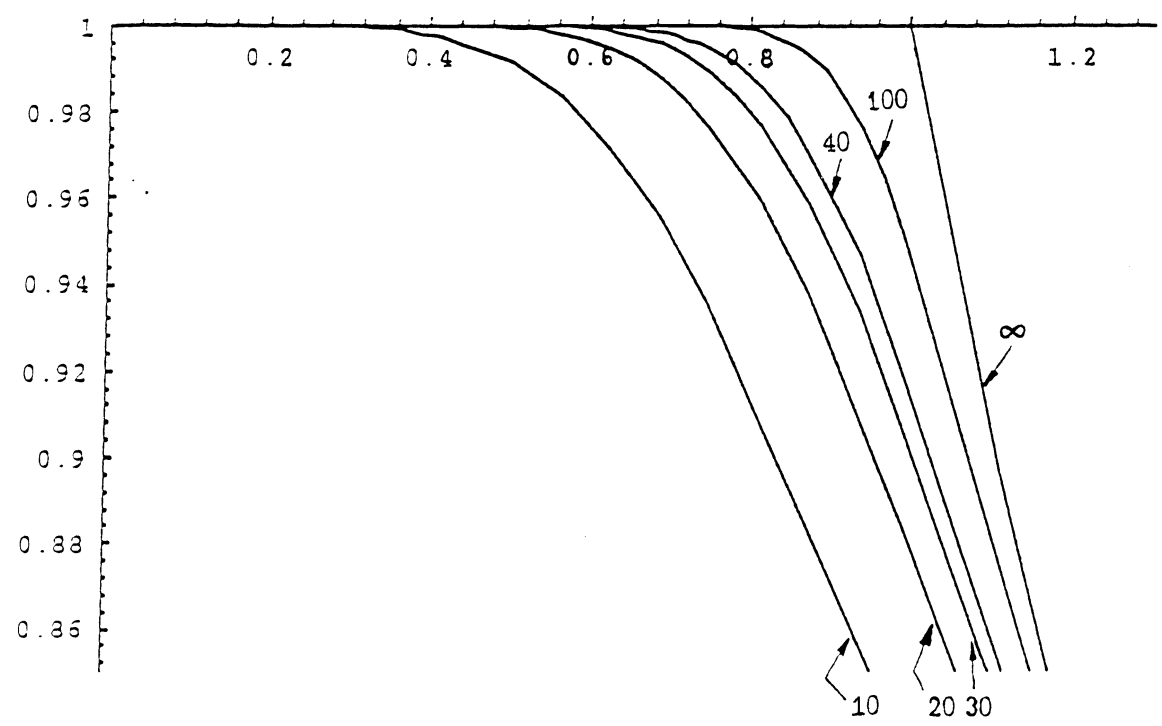

(b)

Fig. 1. (a) Densities of numerical solutions to (5) for $n=10,20,30,40$, and 100 with initial data $c_{j}(0)=\delta_{j, 1}$, and the density of the Leyvraz-Tschudi solution (4), identified by " $\infty$ ", for $a_{j, k}=r_{j} r_{k}$, with $r_{i}=i$. (b) Enlargement of the top left region of (a). 
The proof of this proposition is a straightforward modification of parts (a) and (b) of the proof of Theorem 5.1 of [10] when $\alpha<1$, and of Theorem 2.2 of [17] when $\alpha=1$, and we will omit it.

It must be observed that Proposition 6 says nothing about the uniformity of the convergence, i.e., we cannot infer from it that the density of the approximating solutions will converge to the density of the solution to (1). In fact, this is generally false: For $\alpha \leq 1 / 2$, we know that solutions to (1) conserve densities (see (S1) in the introducion), but the corresponding solutions to the minimally truncated systems (5) continue to show the behaviour indicated in Propositions 4 and 5; i.e., their densities converge to zero as $t \rightarrow \infty$. Thus, Proposition 6 implies nothing about gelation and cannot explain the behaviour of the density plots presented in Figure 1.

Remark 1. For the particular set of conditions used in the experiments of Figure 1, namely, $a_{j, k}=j k$ and $c_{j}(0)=\delta_{j, 1}$, one actually can state a much stronger result than Proposition 6: The theorem in [2] guarantees that $c_{j}^{n}(t)$ converges to $c_{j}(t)$ as $n \rightarrow \infty$, uniformly in $j$ and $t$, for $t \geq 0$. The proof, however, relies on the existence of explicit expressions for the solutions, and hence does not seem to be applicable to more general initial conditions and coagulation coefficients.

\subsection{The Modified System}

The results in Section 2.2, particularly the comparison between the behaviour of the numerical densities to the truncated systems and the exact density (4) to the original problem (1), shown in Figure 1, as well as the observation that in all known cases the post-gel time behaviour of solutions is of the type $\mathcal{O}(1 / t)$ as $t \rightarrow \infty$, suggests that the $1 / t$ decay of geling solutions for large $t$ is probably a significant feature to look at. We now introduce a change of variables that "isolates" the possible $\mathcal{O}(1 / t)$ large time behaviour of solutions to (5). The resulting equations shall be called the Modified System.

Let $t_{0}>0$ be an arbitrary constant, and $c(t)=\left(c_{j}(t)\right)_{1 \leq j \leq n}$ be a solution to (5) in $[0, \infty)$. For all $1 \leq j \leq n$ and all $t \geq 0$, define $x_{j}(t)$ by

$$
x_{j}(t):=\left(1+\frac{t}{t_{0}}\right) c_{j}(t)
$$

thus, the equation for $x_{j}(t)$ is

$$
\left(t+t_{0}\right) \dot{x}_{j}=x_{j}+\frac{1}{2} t_{0} \sum_{k=1}^{j-1} a_{j-k, k} x_{j-k} x_{k}-t_{0} x_{j} \sum_{k=1}^{n} a_{j, k} x_{k}, \quad 1 \leq j \leq n
$$

To transform this nonautonomous system into an autonomous one, we introduce a new (slower) time scale defined by

$$
\tau(t):=\log \left(1+\frac{t}{t_{0}}\right)
$$


and define $y_{j}(\tau)$ by

$$
y_{j}(\tau):=t_{0} x_{j}(t(\tau)),
$$

where $t(\tau)$ is the inverse function of $\tau(t)$, defined on $[0, \infty)$ by $t(\tau)=t_{0}\left(e^{\tau}-1\right)$.

Hence, denoting by $(\cdot)^{\prime}$ the derivative with respect to $\tau$, the initial value problem for (5) becomes, for $1 \leq j \leq n$,

$$
\left\{\begin{array}{l}
y_{j}^{\prime}=y_{j}+\frac{1}{2} \sum_{k=1}^{j-1} a_{j-k, k} y_{j-k} y_{k}-y_{j} \sum_{k=1}^{n} a_{j, k} y_{k}, \\
y_{j}(0)=y_{0 j},
\end{array}\right.
$$

where $y_{0 j}:=t_{0} c_{0 j} \geq 0$, and $c_{0}=\left(c_{0 j}\right)$ is the initial data for (5).

Global existence and uniqueness of solutions to (10) is guaranteed by the above construction and the results about solutions to (5), since $y(\tau)$ is a solution to (10) if and only if $c(t)$ is a solution to (5) and (7)-(9) hold. Likewise, the positivity result in Proposition 2 remains valid for solutions of (10). On the general asymptotic behaviour of solutions to (10) we can note the following:

(i) $y_{j}(\tau) \rightarrow 0$ as $\tau \rightarrow \infty$ if and only if $c_{j}(t) \sim o(1 / t)$ as $t \rightarrow \infty$.

(ii) $y_{j}(\tau)$ is bounded as $\tau \rightarrow \infty$ if and only if $c_{j}(t) \sim \mathcal{O}(1 / t)$ as $t \rightarrow \infty$.

(iii) $y_{j}(\tau)$ is unbounded as $\tau \rightarrow \infty$ if and only if $c_{j}(t)$ decays to zero as $t \rightarrow \infty$ at a slower rate than $1 / t$.

The next proposition shows that only case (ii) occurs.

Proposition 7. Let $y(\tau)=\left(y_{j}(\tau)\right)_{1 \leq j \leq n}$ be a nonzero and nonnegative solution of (10) in $[0, \infty)$. Then $y(\tau)$ is bounded and bounded away from zero.

Proof. It is sufficient to study what happens when $\tau \rightarrow \infty$. To show that $y(\tau)$ is bounded away from zero, simply note that the linearization of (10) about the zero solution gives $y^{\prime}=I_{n} y$ where $I_{n}$ is the $n$-dimensional identity matrix, and so the solution $y=0$ is linearly unstable. To prove that $y(\tau)$ is bounded, consider first the case $j=1$ :

$$
y_{1}^{\prime}=y_{1}-y_{1} \sum_{k=1}^{n} a_{1, k} y_{k} \leq y_{1}-a_{1,1} y_{1}^{2} .
$$

Let $F_{i}(w):=w\left(1-a_{i, i} w\right)$. Then, all positive solutions of $w^{\prime}=F_{1}(w)$ are bounded in $[0, \infty)$ because $w=a_{1,1}^{-1}$ is the global attractor for positive solutions. Let $w_{1}(\cdot)$ be a solution of this equation with $w_{1}(0)=y_{01}>0$. Then $0<y_{1}(\tau) \leq w_{1}(\tau)$ for all $\tau \geq 0$, and thus $y_{1}$ is bounded. Consider now the case $j=2$ :

$$
y_{2}^{\prime}=y_{2}+\frac{1}{2} a_{1,1} y_{1}^{2}-y_{2} \sum_{k=1}^{n} a_{2, k} y_{k} \leq \frac{1}{2} a_{1,1} y_{1}^{2}+F_{2}\left(y_{2}\right) .
$$

Since we have $y_{1}$ bounded, there exists $\kappa_{1}$ dependent on the initial data, such that $a_{1,1} y_{1}^{2} \leq$ $2 \kappa_{1}$, so that

$$
y_{2}^{\prime} \leq \kappa_{1}+F_{2}\left(y_{2}\right)
$$


Since $w=\frac{1+\sqrt{1+4 a_{2,2} \kappa_{1}}}{2 a_{2,2}}$ is the global attractor of positive solutions to $w^{\prime}=\kappa_{1}+F_{2}(w)$, we conclude that $y_{2}(\tau)$ is bounded in $[0, \infty)$. Suppose we have proved boundedness of $y_{1}, \ldots, y_{p-1}$. Then, there exists a constant $\kappa_{p-1}$, dependent of the initial data, such that $\sum_{k=1}^{p-1} a_{p-k, k} y_{p-k} y_{k} \leq 2 \kappa_{p-1}$, and so

$$
y_{p}^{\prime}=y_{p}+\frac{1}{2} \sum_{k=1}^{p-1} a_{p-k, k} y_{p-k} y_{k}-y_{p} \sum_{k=1}^{n} a_{p, k} y_{k} \leq \kappa_{p-1}+F_{p}\left(y_{p}\right) .
$$

Repeating the previous argument, we conclude that $y_{p}(\tau)$ is bounded, and proceeding up to $j=n$ proves the result.

\section{Equilibria of the Modified System and Geling Solutions}

From this section onwards, we will consider the following hypothesis:

(H1) $a_{j, k}=r_{j} r_{k}$ for all $j$ and $k$, where $\left(r_{j}\right)$ is a strictly increasing positive sequence.

Remember also that, for any $\alpha>0,\lfloor\alpha\rfloor$ denotes the integer part of $\alpha$, i.e., $\lfloor\alpha\rfloor=$ $\max \{p \in \mathbb{N}: p \leq \alpha\}$.

In the next proposition we study the equilibria of (10).

Proposition 8. Assume (H1). For all $n \in \mathbb{N}$, the modified system (10) has $n+1$ nonnegative equilibria, denoted by $\bar{y}^{(L)}$, or by $\bar{y}^{(L, n)}$ whenever we need to state explicitly the value of $n$, with $L=0,1, \ldots, n$, which are the following:

for $L=0: \bar{y}^{(0)}=\underbrace{(0,0, \ldots, 0)}_{n \text { components }}$;

for $L \geq 1: \bar{y}^{(L)}=\left(\bar{y}_{j}^{(L)}\right)_{1 \leq j \leq n}$, where

$$
\bar{y}_{j}^{(L)}=0 \quad \text { if } \quad j \notin L \cdot \mathbb{N} \text {; }
$$

and

$$
\bar{y}_{j L}^{(L)}=Q_{j}^{(L)}\left(\bar{y}_{L}^{(L)}\right)^{j} \quad \text { for all } \quad j=2, \ldots,\lfloor n / L\rfloor,
$$

with $Q_{j}^{(L)}=\beta_{j}^{(L)} r_{L}^{j} r_{j L}^{-1}$ and

$$
\left\{\begin{array}{l}
\beta_{1}^{(L)}=1, \\
\beta_{j}^{(L)}=\frac{1}{2\left(r_{L}^{-1}-r_{j L}^{-1}\right)} \sum_{k=1}^{j-1} \beta_{j-k}^{(L)} \beta_{k}^{(L)}, \quad 2 \leq j \leq\lfloor n / L\rfloor,
\end{array}\right.
$$

and $\bar{y}_{L}^{(L)}$ is the only positive zero of the polynomial defined by

$$
P_{L, n}(x):=\sum_{j=1}^{\lfloor n / L\rfloor} \beta_{j}^{(L)}\left(r_{L} x\right)^{j}-r_{L}^{-1}
$$


Proof. The existence of $\bar{y}^{(0)}$ is obvious. We start with the case $L=1$. From the convention that the first sum in the right-hand side of (10) is zero for $j=1$, we obtain

$$
\left\{\begin{array}{l}
\bar{y}_{1}^{(1)}-r_{1} \bar{y}_{1}^{(1)} \sum_{k=1}^{n} r_{k} \bar{y}_{k}^{(1)}=0, \\
\bar{y}_{j}^{(1)}+\frac{1}{2} \sum_{k=1}^{j-1} r_{j-k} r_{k} \bar{y}_{j-k}^{(1)} \bar{y}_{k}^{(1)}-r_{j} \bar{y}_{j}^{(1)} \sum_{k=1}^{n} r_{k} \bar{y}_{k}^{(1)}=0, \quad 2 \leq j \leq n .
\end{array}\right.
$$

Since by hypothesis $\bar{y}_{1}^{(1)}>0$, we must have

$$
\sum_{k=1}^{n} r_{k} \bar{y}_{k}^{(1)}=r_{1}^{-1}
$$

For $2 \leq j \leq n$, the components $\bar{y}_{j}^{(1)}$ must satisfy

$$
\bar{y}_{j}^{(1)}=\frac{r_{1}}{2\left(r_{j}-r_{1}\right)} \sum_{k=1}^{j-1} r_{j-k} r_{k} \bar{y}_{j-k}^{(1)} \bar{y}_{k}^{(1)},
$$

and the conclusion readily follows.

Now assume $L \geq 2$, and let $\bar{y}^{(L)}$ be the equilibria of (10) such that $\bar{y}_{j}^{(L)}=0$ for all $j \leq L-1$ and $\bar{y}_{L}^{(L)}>0$. Then, $\sum_{k=1}^{j-1} r_{j-k} r_{k} \bar{y}_{j-k}^{(L)} \bar{y}_{k}^{(L)}=0$ for all $1 \leq j \leq 2 L-1$, because $\min _{1 \leq k \leq j-1}\{j-k, k\} \leq j / 2 \leq \frac{1}{2}(2 L-1)<L$, and so in each of the products $\bar{y}_{j-k}^{(L)} \bar{y}_{k}^{(L)}$ one of the terms is zero. This means that, for $L \leq j \leq 2 L-1$, the equations defining the equilibria are

$$
\bar{y}_{j}^{(L)}-r_{j} \bar{y}_{j}^{(L)} \sum_{k=L}^{n} r_{k} \bar{y}_{k}^{(L)}=0 .
$$

Using $\bar{y}_{L}^{(L)}>0$ and $j=L$ in (15), we obtain

$$
\sum_{j=L}^{n} r_{j} \bar{y}_{j}^{(L)}=r_{L}^{-1}
$$

Substituting this result into (15), we can write

$$
\bar{y}_{j}^{(L)}\left(1-r_{j} r_{L}^{-1}\right)=0 \quad \text { for } \quad L+1 \leq j \leq 2 L-1,
$$

which implies $\bar{y}_{j}^{(L)}=0$ for these values of $j$. For $2 L \leq j \leq n$, we must have

$$
\bar{y}_{j}^{(L)}+\frac{1}{2} \sum_{k=L}^{j-L} r_{j-k} r_{k} \bar{y}_{j-k}^{(L)} \bar{y}_{k}^{(L)}-r_{j} \bar{y}_{j}^{(L)} \sum_{k=L}^{n} r_{k} \bar{y}_{k}^{(L)}=0 .
$$

Thus, using (16),

$$
\bar{y}_{j}^{(L)}=\frac{1}{2\left(r_{j} r_{L}^{-1}-1\right)} \sum_{k=L}^{j-L} r_{j-k} r_{k} \bar{y}_{j-k}^{(L)} \bar{y}_{k}^{(L)} .
$$


Now we prove that $\bar{y}_{j}^{(L)}$ given by (17) is nonzero if and only if $j \in L \cdot \mathbb{N}$. Consider $j=p L$ for some $p \geq 2$, and suppose $\bar{y}_{k L}^{(L)}>0$ for all $k \in\{1, \ldots, p-1\}$, then

$$
\begin{aligned}
\bar{y}_{p L}^{(L)} & =\frac{1}{2\left(r_{p L} r_{L}^{-1}-1\right)} \sum_{k=L}^{(p-1) L} r_{p L-k} r_{k} \bar{y}_{p L-k}^{(L)} \bar{y}_{k}^{(L)} \\
& \geq \frac{1}{2\left(r_{p L} r_{L}^{-1}-1\right)} r_{(p-1) L} r_{L} \bar{y}_{(p-1) L}^{(L)} \bar{y}_{L}^{(L)} \\
& >0 .
\end{aligned}
$$

Hence, by induction, $\bar{y}_{j}^{(L)}>0$ if $j \in L \cdot \mathbb{N}$. Suppose now $j$ is not a multiple of $L$. For $1 \leq j \leq L-1$ and $L+1 \leq j \leq 2 L-1$, we already know that $\bar{y}_{j}^{(L)}=0$. Define $\mathcal{N}_{p}:=\{j \leq p L-1: j \notin L \cdot \mathbb{N}\}$. To prove that if $\bar{y}_{j}^{(L)}=0$ for $j \in \mathcal{N}_{p}$ then the same happens for $j \in \mathcal{N}_{p+1}$, it is only necessary to observe that, for $k=L, \ldots, j-L$, either $k$ or $j-k$ is not a multiple of $L$ because if both were multiples of $L$ then $j=(j-k)+k$ also would be. But then, using (17) and the fact that $j-L \in \mathcal{N}_{p}$ for all $j \in \mathcal{N}_{p+1}$, we obtain the result.

Easy algebraic manipulations allow us to write the components of the equilibria $\bar{y}^{(L)}$ in the form (11)-(14), concluding the proof.

Remark 2. It is interesting to observe the structure of the equilibria given in Proposition 8 , namely, the fact that the only nonzero components of $\bar{y}^{(L)}$ are the $\bar{y}_{j}^{(L)}$ for which $j$ is a multiple of $L$ :

$$
\begin{aligned}
& \bar{y}^{(1)}: \quad(*, *, *, *, *, *, *, *, *, \ldots, *, *), \\
& \bar{y}^{(2)}: \quad(0, *, 0, *, 0, *, 0, *, 0, \ldots \ldots) \text {, } \\
& \bar{y}^{(3)}: \quad(0,0, *, 0,0, *, 0,0, *, \ldots \ldots) \text {, } \\
& \bar{y}^{(4)}: \quad(0,0,0, *, 0,0,0, *, 0, \ldots \ldots) \text {, } \\
& \bar{y}^{(5)}: \quad(0,0,0,0, *, 0,0,0,0, \ldots \ldots) \text {, } \\
& \begin{array}{ll}
\bar{y}^{(n-1)}: & (0,0,0,0,0,0,0,0,0, \ldots, *, 0), \\
\bar{y}^{(n)}: & (0,0,0,0,0,0,0,0,0, \ldots, 0, *),
\end{array}
\end{aligned}
$$

where the symbol $*$ simply indicates that the component of the equilibria in that location is different from zero (and given as stated in Proposition 8).

We now show that the equilibrium $\bar{y}^{(1)}$ of $(10)$ is related closely to the Leyvraz solution [18], referred to in (P5). This is not surprising: Looking at the derivation of the Leyvraz solution in [18], we notice that the Leyvraz sequence $\left(\alpha_{j}\right)$ is given by

$$
\left\{\begin{array}{l}
\alpha_{j}=\frac{1}{2\left(r_{j} r_{1}^{-1}-1\right)} \sum_{k=1}^{j-1} r_{j-k} r_{k} \alpha_{j-k} \alpha_{k}, \quad j \geq 2, \\
\sum_{k=1}^{\infty} r_{k} \alpha_{k}-r_{1}^{-1}=0
\end{array}\right.
$$


Thus, for all $j \geq 2$, we have $\alpha_{j}=Q_{j}^{(1)} \alpha_{1}^{j}$ with $Q_{j}^{(1)}$ defined as in Proposition 8 , and $\alpha_{1}$ the only positive zero of

$$
P_{1}(x):=\sum_{j=1}^{\infty} r_{j} Q_{j}^{(1)} x^{j}-r_{1}^{-1} .
$$

Hence, it is natural to expect that, in some convenient sense, the equilibrium $\bar{y}^{(1, n)}$ of the $n$-dimensional modified system (10) converges to the Leyvraz solution of (1) as $n \rightarrow \infty$.

Proposition 9. Assume (H1). Let $\bar{y}^{(1, n)}$ be the equilibrium of the $n$-dimensional modified system (10) defined by (11)-(14) with $L=1$. Let $\left(\alpha_{j}\right)$ be the Leyvraz sequence. Then, for each fixed $j, \bar{y}_{j}^{(1, n)} \longrightarrow \alpha_{j}$ and

$$
\sum_{j=1}^{n} r_{j} \bar{y}_{j}^{(1, n)} \longrightarrow \sum_{j=1}^{\infty} r_{j} \alpha_{j} \quad \text { as } \quad n \rightarrow \infty .
$$

Proof. Consider the sequence $\left(\bar{y}^{(1, n)}\right)_{n \geq 1}$. By Proposition 8 we conclude that, for all $n$, $P_{1, n}\left(\bar{y}^{(1, n)}\right)=0=P_{1}\left(\alpha_{1}\right)$, and thus

$$
\lim _{n \rightarrow \infty} \sum_{j=1}^{n} r_{j} \bar{y}_{j}^{(1, n)}=r_{1}^{-1}=\sum_{j=1}^{\infty} r_{j} \alpha_{j} .
$$

For the pointwise convergence of $\left(\bar{y}^{(1, n)}\right)_{n \geq 1}$, we need to remember that for each $n$ the only positive solution of $P_{1, n}(x)=0$ is $\bar{y}_{1}^{(1, n)}$. Since $r_{k} Q_{k}^{(1)}>0$ for all $k \geq 1$, the sequence $\left(\bar{y}_{1}^{(1, n)}\right)_{n \geq 1}$ is strictly decreasing and bounded below by zero, and thus it converges to some $\bar{y}_{1}^{*} \geq 0$. It is not difficult to prove that we must have $\bar{y}_{1}^{*}=\alpha_{1}$, and the result for the other components follows easily from the relations of the $j^{\text {th }}$ and the $1^{\text {st }}$ components of both $\alpha$ and $\bar{y}^{(L, n)}$. We will omit further details.

This result suggests that, for each $L \geq 1$, there should exist a Leyvraz-type sequence $\left(\alpha_{j}^{(L)}\right)$ such that $c_{j}(t)=\alpha_{j}^{(L)}\left(t+t_{0}\right)^{-1}$ is a solution of (1) and that, as $n \rightarrow \infty$, and for all $j$ and $L, \bar{y}_{j}^{(L, n)} \longrightarrow \alpha_{j}^{(L)}$, where $\bar{y}_{j}^{(L, n)}$ is the $j^{\text {th }}$ component of the equilibrium solution of (10) given by (11)-(14). In fact, the following holds true:

Proposition 10. Assume (H1), and $r_{j} \geq A j^{\alpha}$ with $A>0$ and $\alpha>1 / 2$ constants. Then, for each $L \in \mathbb{N}$, there exists a nonnegative sequence $\left(\alpha_{j}^{(L)}\right)_{j \in \mathbb{N}}$ satisfying $\alpha_{j}^{(L)}=0$ if $j \notin$ $L \cdot \mathbb{N}$ and $\sum_{j=1}^{\infty} j \alpha_{j}^{(L)}<\infty$, and such that $c=\left(c_{j}^{(L)}\right)_{j \in \mathbb{N}}$ defined by $c_{j}^{(L)}:=\left(t+t_{0}\right)^{-1} \alpha_{j}^{(L)}$ is a solution of $(1)$ in $[0, \infty)$ with initial data $c_{0 j}=\alpha_{j}^{(L)} t_{0}^{-1}$ with $t_{0}>0$ an arbitrary constant. Furthermore, for all $1 \leq L \leq n$, the equilibria $\bar{y}^{(L, n)}$ of the $n$-dimensional modified system, defined by (11)-(14), satisfy $\bar{y}_{j}^{(L, n)} \longrightarrow \alpha_{j}^{(L)}$ and $\sum_{j=1}^{n} r_{j} \bar{y}_{j}^{(L, n)} \longrightarrow$ $\sum_{j=1}^{\infty} r_{j} \alpha_{j}^{(L)}$ as $n \rightarrow \infty$.

The proof of this result will be omitted since it is essentially just a change of notation in the proof given, for the case $L=1$, by Leyvraz in [18] and, for the last part, of the proof of Proposition 9 above. 


\section{Dynamics of the Modified System}

We now turn to the study of the dynamic behaviour of nonnegative solutions to the modified system (10), both analytically and numerically.

The positivity properties of solutions stated in Proposition 2 imply, in particular, that the sets $\mathcal{S}_{M}$ defined by

$$
\begin{aligned}
& \mathcal{S}_{1}:=\left\{y \in \mathbb{R}^{n}: y_{1}>0, \quad y_{j} \geq 0 \text { for } 2 \leq j \leq n\right\}, \\
& \mathcal{S}_{n}:=\left\{y \in \mathbb{R}^{n}: y_{n}>0, \quad y_{j}=0 \text { for all } 1 \leq j \leq n-1\right\},
\end{aligned}
$$

and, for $1<M<n$,

$$
\mathcal{S}_{M}:=\left\{y \in \mathbb{R}^{n}: y_{1}=\cdots=y_{M-1}=0, y_{M}>0, y_{j} \geq 0 \text { for } M+1 \leq j \leq n\right\},
$$

are positively invariant for the flow defined by (10), and its closure $\overline{\mathcal{S}_{M}}$ is also positively invariant. The usefulness of these sets is due to this invariance property, to the fact that $\mathcal{S}_{M+1} \subset \partial \mathcal{S}_{M} \subset \overline{\mathcal{S}_{M}}$ for all $M \geq 1$, and what is rather important for our purposes, to the fact that $\mathcal{S}_{M}$ is the "natural" set associated with the equilibrium $\bar{y}^{(M)}$, in the sense that, for each $M, \bar{y}^{(M)}$ is the only equilibrium point of (10) lying in $\mathcal{S}_{M}$. Furthermore, it is clear that $\bar{y}^{(L)} \in \overline{\mathcal{S}_{M}}$ for all $L \geq M \geq 1$.

These properties of the sets $\mathcal{S}_{M}$, and their relations with the equilibria, mean that it is possible to restrict the modified system (10) to any of the sets $\overline{\mathcal{S}_{M}}$ and to study the stability properties, relative to this restriction, of the equilibria $\bar{y}^{(L)}$ for $L \geq M$.

We start by investigating the linear stability properties of the equilibria.

\subsection{General Linear Stability Properties of Equilibria}

Let $1 \leq M \leq L \leq n$ and $\mu=\lfloor n / L\rfloor$. Let $\mathcal{K}^{(L, n)}$ be the $(\mu \times \mu)$ matrix defined by

$$
\mathcal{K}^{(L, n)}:=\left[\begin{array}{cccc}
\alpha_{L, L}^{(n)} & \alpha_{L, 2 L}^{(n)} & \cdots & \alpha_{L, \mu L}^{(n)} \\
\alpha_{2 L, L}^{(n)} & \alpha_{2 L, 2 L}^{(n)} & \cdots & \alpha_{2 L, \mu L}^{(n)} \\
\vdots & \vdots & & \vdots \\
\alpha_{\mu L, L}^{(n)} & \alpha_{\mu L, 2 L}^{(n)} & \cdots & \alpha_{\mu L, \mu L}^{(n)},
\end{array}\right]
$$

where

$$
\alpha_{j L, i L}^{(n)}:= \begin{cases}-r_{i L} r_{j L} \bar{y}_{j L}^{(L, n)}, & \text { if } i>j, \\ 1-r_{j L} r_{L}^{-1}-r_{j L}^{2} \bar{y}_{j L}^{(L, n)}, & \text { if } i=j, \\ -r_{i L} r_{j L} \bar{y}_{j L}^{(L, n)}+r_{i L} r_{(j-i) L} \bar{y}_{(j-i) L}^{(L, n)}, & \text { if } i<j .\end{cases}
$$

Finally, we denote by $\sigma_{\mathcal{K}}$ the spectrum of the matrix $\mathcal{K}^{(L, n)}$, and define the following sets: $\sigma_{+}:=\left\{1-r_{j} r_{L}^{-1}: M \leq j \leq L-1\right\}$ (with $\sigma_{+}=\emptyset$ if $L=1$ ) and $\sigma_{-}:=$ $\left\{1-r_{j} r_{L}^{-1}: L+1 \leq j \leq n, j \notin L \cdot \mathbb{N}\right\}$ (with $\sigma_{-}=\emptyset$ if $L=n$ ).

With this notation, we have the following.

Proposition 11. Assume (HI). Let $1 \leq M \leq L \leq n$. Let $\mathcal{A}^{(L, M)}$ be the matrix of the linearization about $\bar{y}^{(L)}$ of the restriction of the modified system (10) to $\overline{\mathcal{S}_{M}}$, and denote by $\sigma_{\mathcal{A}}$ the spectrum of $\mathcal{A}^{(L, M)}$. Then, $\sigma_{\mathcal{A}}=\sigma_{+} \cup \sigma_{-} \cup \sigma_{\mathcal{K}}$. 
Proof. For each $1 \leq j \leq n$, let

$$
f_{j}(y):=y_{j}+\frac{1}{2} \sum_{k=1}^{j-1} r_{j-k} r_{k} y_{j-k} y_{k}-r_{j} y_{j} \sum_{k=1}^{n} r_{k} y_{k},
$$

and let $\tilde{f}_{j}:=f_{j}\left\lceil\overline{\mathcal{S}_{M}}\right.$ denote the restriction of $f_{j}$ to $\overline{\mathcal{S}_{M}}$. Then, for all $y=\left(0,0, \ldots, 0, y_{M}\right.$, $\left.y_{M+1}, \ldots, y_{n}\right) \in \overline{\mathcal{S}_{M}}$, we can write

$$
\tilde{f}_{j}(y)= \begin{cases}0, & \text { if } 1 \leq j \leq M-1, \\ y_{j}-r_{j} y_{j} \sum_{k=M}^{n} r_{k} y_{k}, & \text { if } M \leq j \leq 2 M-1, \\ y_{j}+\frac{1}{2} \sum_{k=M}^{j-M} r_{j-k} r_{k} y_{j-k} y_{k}-r_{j} y_{j} \sum_{k=M}^{n} r_{k} y_{k}, & \text { if } 2 M \leq j \leq n .\end{cases}
$$

We shall consider $\tilde{f}=\left(\tilde{f}_{j}\right)$ as a function defined in $\mathbb{R}_{0}^{(n-M+1)+}$ with values in $\mathbb{R}_{0}^{(n-M+1)+}$ by identifying the points $\left(0,0, \ldots, 0, y_{M}, y_{M+1}, \ldots, y_{n}\right) \in \overline{\mathcal{S}_{M}}$ and $\left(y_{M}, y_{M+1}, \ldots, y_{n}\right) \in$ $\mathbb{R}_{0}^{(n-M+1)+}$ and disregarding the identically zero components of $\tilde{f}$ with $1 \leq j \leq M-1$. With this identification, let $\xi=\left(\xi_{M}, \ldots, \xi_{n}\right)$ and consider the linearization of $\tilde{f}$ about $\xi$. For $M \leq j \leq 2 M-1$, we have

$$
\frac{\partial \tilde{f}_{j}}{\partial y_{i}}(\xi)= \begin{cases}1-r_{j} \sum_{k=M}^{n} r_{k} \xi_{k}-r_{j}^{2} \xi_{j}, & \text { if } i=j, \\ -r_{i} r_{j} \xi_{j}, & \text { if } i \neq j,\end{cases}
$$

and for $2 M \leq j \leq n$,

$$
\frac{\partial \tilde{f}_{j}}{\partial y_{i}}(\xi)= \begin{cases}1-r_{j} \sum_{k=M}^{n} r_{k} \xi_{k}-r_{j}^{2} \xi_{j}, & \text { if } i=j, \\ -r_{i} r_{j} \xi, & \text { if } j-M<i<j \text { or } i>j, \\ -r_{i} r_{j} \xi_{j}+r_{j-i} r_{i} \xi_{j-i}, & \text { if } i \leq j-M .\end{cases}
$$

Take now $\xi=\bar{y}^{(L, n)}$ with $L \geq M$. From (11), $\bar{y}_{j}^{(L, n)}=0$ if $j$ is not a multiple of $L$, and by (16) we have $\sum_{k=M}^{n} r_{k} \bar{y}_{k}^{(L, n)}=\sum_{k=1}^{\mu} r_{k L} \bar{y}_{k L}^{(L, n)}=r_{L}^{-1}$. This allows us to simplify considerably the Jacobian matrix of $\tilde{f}$. To be able to compare easily the results of the linearizations of $f\left\lceil\overline{\mathcal{S}}_{M}\right.$ about $\bar{y}^{(L, n)}$ for different values of $L$ and $M$, it is convenient to consider the subscripts of the elements of the $(n-M+1) \times(n-M+1)$ Jacobian matrices as running from $M$ to $n$, instead of from 1 to $n-M+1$, as is the convention. With this consideration, the Jacobian matrix $\mathcal{A}^{(L, M)}$ has the structure exemplified in Figure 2.

The spectrum of $\mathcal{A}^{(L, M)}$ is now obtained easily by the Laplace expansion by minors of the determinant of $\mathcal{A}^{(L, M)}-\lambda I_{n-M+1}$ along the rows with $j \notin L \cdot \mathbb{N}$ :

$$
\operatorname{det}\left(\mathcal{A}^{(L, M)}-\lambda I_{n-M+1}\right)=\operatorname{det}\left(\mathcal{K}^{(L, n)}-\lambda I_{\mu}\right) \prod_{\substack{M \leq \leq \leq n \\ j \notin L: \mathbb{N}}}\left(1-\frac{r_{j}}{r_{L}}-\lambda\right) .
$$




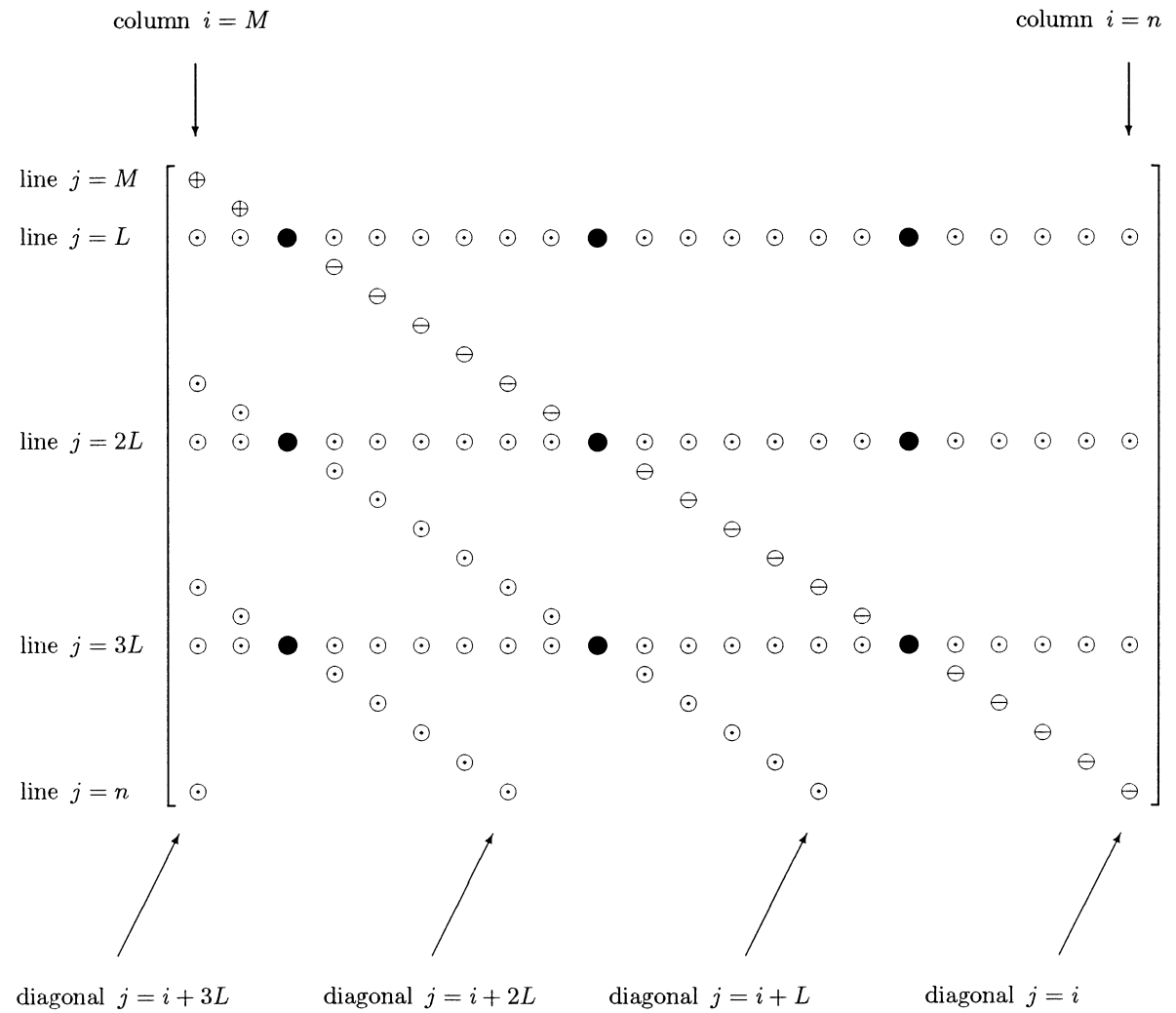

Fig. 2. Structure of the Jacobian matrix $\mathcal{A}^{(L, M)}$. (Example for the case $M=5, L=7, n=26$, in which case $\mu=\lfloor 26 / 7\rfloor=3$.) The matrix elements whose positions are not explicitly marked are zero. For the nonzero elements of the matrix, the following notation is used: $\oplus$ are elements belonging to $\sigma_{+}, \ominus$ are elements belonging to $\sigma_{-}, \odot$ are elements of $\mathcal{K}^{(L, n)}$, and $\odot$ are other nonzero elements. The values of all these nonzero elements are obtained from expressions (21) and (22) computed at $\xi=\bar{y}^{(L, n)}$.

Observing that the terms of the product with $M \leq j \leq L-1$ are precisely the elements of $\sigma_{+}$(signalled by $\oplus$ in Figure 2) and the terms with $j \geq L+1$, but not multiples of $L$, are the elements of $\sigma_{-}\left(\ominus\right.$ in Figure 2), expression (23) says that $\sigma_{\mathcal{A}}=\sigma_{+} \cup \sigma_{-} \cup \sigma_{\mathcal{K}}$, as we wanted to prove.

The study of the local linear stability of $\bar{y}^{(L, n)}$ in $\overline{\mathcal{S}_{M}}$ is now reduced to the study of $\sigma_{\mathcal{K}}$, the spectrum of $\mathcal{K}^{(L, n)}$.

Remark 3. From assumption (H1), we can conclude immediately that

(i) all the $L-M$ elements of $\sigma_{+}$are positive.

(ii) all the $n-L-\mu+1$ elements of $\sigma_{-}$are negative. 
Using (H1), (19), (20), and Proposition 8, we conclude that

$$
\begin{aligned}
\operatorname{tr} \mathcal{K}^{(L, n)} & =\sum_{j=1}^{\mu}\left(1-r_{j L} r_{L}^{-1}-r_{j L}^{2} \bar{y}_{j L}^{(L, n)}\right)=\mu-r_{L}^{-1} \sum_{j=1}^{\mu} r_{j L}-\sum_{j=1}^{\mu} r_{j L}^{2} \bar{y}_{j L}^{(L, n)} \\
& <\mu-r_{L}^{-1} \sum_{j=1}^{\mu} r_{L}-\sum_{j=1}^{\mu} r_{j L}^{2} \bar{y}_{j L}^{(L, n)}=-\sum_{j=1}^{\mu} r_{j L}^{2} \bar{y}_{j L}^{(L, n)} \\
& <-r_{L} \sum_{j=1}^{\mu} r_{j L} \bar{y}_{j L}^{(L, n)}=-1,
\end{aligned}
$$

and thus

(iii) at least one of the eigenvalues of $\sigma_{\mathcal{K}}$ has a negative real part.

Despite numerous attempts, it has not been possible to fully describe $\sigma_{\mathcal{K}}$ in the general case. For general strictly increasing positive sequences $\left(r_{j}\right)$, the best result we could prove is the following.

Proposition 12. With the assumptions of Proposition 11, we have $\operatorname{Re}\left(\sigma_{\mathcal{K}}\right)<0$ for $\mu=1$ and $\mu=2$.

Proof. Both cases are trivial: If $\mu=1$, then $\mathcal{K}^{(L, n)}$ has only one element, which is $-r_{L}^{2} \bar{y}_{L}^{(L, n)}<0$. For $\mu=2$, the matrix $\mathcal{K}^{(L, n)}$ is a $2 \times 2$ matrix with negative trace (by Remark 3) and

$$
\operatorname{det} \mathcal{K}^{(L, n)}=\left(r_{2 L}-r_{L}\right) r_{L} \bar{y}_{L}^{(L, n)}+r_{2 L} r_{L}^{3}\left(\bar{y}_{L}^{(L, n)}\right)^{2}>0,
$$

from which the result follows.

Thus we have the following local stability result.

Proposition 13. With the assumptions of Proposition 11, denote by $W_{s}^{(L, M)}$ and $W_{u}^{(L, M)}$ the stable and unstable manifolds of $\bar{y}^{(L, n)}$ in $\overline{\mathcal{S}_{\mathrm{M}}}$, respectively. Let $\mu=1$ or $\mu=2$. Then,

$$
\begin{aligned}
& \operatorname{dim} W_{u}^{(L, M)}=L-M, \\
& \operatorname{dim} W_{s}^{(L, M)}=n-L+1 .
\end{aligned}
$$

In particular, $\bar{y}^{(L, n)}$ is locally exponentially asymptotically stable in $\mathcal{S}_{L}$.

For $\mu \geq 3$ we were unable, so far, to prove that all elements of $\sigma_{\mathcal{K}}$ have negative real parts for a general sequence $\left(r_{j}\right)$, although all available numerical evidence suggests this is indeed true. The only case for which we could complete the analysis of $\sigma_{\mathcal{K}}$ is when $r_{j}=j$, and this will be presented in Section 4.2. We next present some of the numerical evidence that supports the following conjecture.

Conjecture 1. Proposition 12 holds true for all values of $\mu$. 
Table 2. Spectra of $\mathcal{K}^{(L, n)}$ for $r_{j}=1+j^{3 / 5}, L=1,2,5$, and $\mu=3,5,6,10$.

\begin{tabular}{|c|c|c|c|c|}
\hline$L$ & $\mu=3$ & $\mu=5$ & $\mu=6$ & $\mu=10$ \\
\hline 1 & $\begin{array}{l}-1 \\
-0.481+0.087 i \\
-0.481-0.087 i\end{array}$ & $\begin{array}{l}-1 \\
-0.813 \\
-0.652 \\
-0.555+0.156 i \\
-0.555-0.156 i\end{array}$ & $\begin{array}{l}-1 \\
-0.965 \\
-0.814 \\
-0.654 \\
-0.584+0.180 i \\
-0.584-0.180 i\end{array}$ & $\begin{array}{l}-1.491 \\
-1.369 \\
-1.241 \\
-1.107 \\
-1 \\
-0.966 \\
-0.818 \\
-0.660 \\
-0.675+0.249 i \\
-0.675-0.249 i\end{array}$ \\
\hline 2 & $\begin{array}{l}-1 \\
-0.573+0.102 i \\
-0.573-0.102 i\end{array}$ & $\begin{array}{l}-1 \\
-0.980 \\
-0.787 \\
-0.657+0.187 i \\
-0.657-0.187 i\end{array}$ & $\begin{array}{l}-1.163 \\
-1 \\
-0.981 \\
-0.791 \\
-0.689+0.216 i \\
-0.689-0.216 i\end{array}$ & $\begin{array}{l}-1.796 \\
-1.649 \\
-1.496 \\
-1.334 \\
-1.164 \\
-1 \\
-0.987 \\
-0.803 \\
-0.788+0.298 i \\
-0.788+0.298 i\end{array}$ \\
\hline 5 & $\begin{array}{l}-1 \\
-0.681+0.120 i \\
-0.681-0.120 i\end{array}$ & $\begin{array}{l}-1.178 \\
-1 \\
-0.947 \\
-0.775+0.222 i \\
-0.775-0.222 i\end{array}$ & $\begin{array}{l}-1.398 \\
-1.179 \\
-1 \\
-0.954 \\
-0.811+0.257 i \\
-0.811-0.257 i\end{array}$ & $\begin{array}{l}-2.159 \\
-1.982 \\
-1.798 \\
-1.604 \\
-1.399 \\
-1.187 \\
-1 \\
-0.975 \\
-0.919+0.355 i \\
-0.919+0.355 i\end{array}$ \\
\hline
\end{tabular}

In Table 2 we present the numerically computed spectra of $\mathcal{K}^{(L, n)}$ for a nonhomogeneous sequence $\left(r_{j}\right)$ and several values of $L$ and $n$. In Table 3 we present the numerically computed spectra of $\mathcal{K}^{(1, \mu)}$ for several values of $\mu$ and for two different homogeneous sequences $\left(r_{j}\right)$.

The distinction, made explicit in Tables 2 and 3, between homogeneous and nonhomogeneous sequences $\left(r_{j}\right)$ is due to the fact that, as the next proposition shows, for homogeneous sequences the linearizations about $\bar{y}^{(L, n)}$ and $\bar{y}^{(1, \mu)}$, with $\mu=\lfloor n / L\rfloor$, are the same, and so we need only to study the spectra of $\mathcal{K}^{(1, \mu)}$.

Proposition 14. Let $1 \leq L \leq n, \mu=\lfloor n / L\rfloor$, and suppose that $\left(r_{j}\right)$ is a homogeneous sequence, i.e., $r_{j}=A j^{\alpha}$ for some positive constants $A$ and $\alpha$. Then, $\mathcal{K}^{(L, n)}=\mathcal{K}^{(1, \mu)}$.

Proof. In order to relate $\mathcal{K}^{(L, n)}$ and $\mathcal{K}^{(1, \mu)}$, we need to relate the equilibria $\bar{y}^{(L, n)}$ and 
Table 3. Spectra of $\mathcal{K}^{(1, \mu)}$ for $r_{j}=j$ and $r_{j}=1.414 j^{3 / 4}$, with $\mu=3,5,6,10$.

\begin{tabular}{cllll}
\hline$r_{j}$ & $\mu=3$ & $\mu=5$ & $\mu=6$ & $\mu=10$ \\
\hline$j$ & -1 & -1 & -1 & -1 \\
& -1.691 & -2 & -2 & -2 \\
& -2 & -2.199 & -2.419 & -3 \\
& -3 & -3 & -3.167 \\
& -4 & -4 & -4 \\
& & -5 & -5 \\
& & & -6 \\
& & & -7 \\
& & & & -8 \\
$1.414 j^{3 / 4}$ & -1 & -1 & -1 & -9 \\
& $-1.230+0.153 i$ & -1.813 & -1.796 & -1 \\
& $-1.230-0.153 i$ & -2.343 & -2.342 & -1.696 \\
& $-1.403+0.230 i$ & -2.834 & -2.329 \\
& $-1.403-0.230 i$ & $-1.480+0.338 i$ & -3.303 \\
& & $-1.480-0.338 i$ & -3.756 \\
& & & & -4.196 \\
& & & & -4.623 \\
& & & & $-1.753+0.456 i$ \\
& & & & $-1.753-0.456 i$ \\
\hline
\end{tabular}

$\bar{y}^{(1, \mu)}$. From (13), we have

$$
\beta_{j}^{(L)}=L^{\alpha} \frac{A j^{\alpha}}{2\left(j^{\alpha}-1\right)} \sum_{k=1}^{j-1} \beta_{j-k}^{(L)} \beta_{k}^{(L)},
$$

and

$$
\beta_{j}^{(1)}=\frac{A j^{\alpha}}{2\left(j^{\alpha}-1\right)} \sum_{k=1}^{j-1} \beta_{j-k}^{(1)} \beta_{k}^{(1)},
$$

and since $\beta_{1}^{(L)}=\beta_{1}^{(1)}=1$, we immediately conclude that $\beta_{j}^{(L)}=L^{(j-1) \alpha} \beta_{j}^{(1)}$. From (14), we have that $x=r_{L} \bar{y}_{L}^{(L, n)}$ is the only positive solution of $\sum_{k=1}^{\mu} \beta_{k}^{(L)} x^{k}=r_{L}^{-1}$ and so, using the relation between the $\beta_{k}^{(L)}$ and the $\beta_{k}^{(1)}$ just obtained and the form of $r_{j}$, it is also the only positive solution of $\sum_{k=1}^{\mu} \beta_{k}^{(1)}\left(L^{\alpha} x\right)^{k}=A^{-1}$. But since $r_{1}=A$, the last equation has $L^{\alpha} x=r_{1} \bar{y}_{1}^{(1, \mu)}$ as its only positive solution, and thus we must have $L^{\alpha} r_{L} \bar{y}_{L}^{(L, n)}=r_{1} \bar{y}_{1}^{(1, \mu)}$. The relation between the other components now follow easily from (12) and the relations above:

$$
r_{j L} \bar{y}_{j L}^{(L, n)}=\beta_{j}^{(L)}\left(r_{L} \beta_{L}^{(L, n)}\right)^{j}=L^{-\alpha} \beta_{j}^{(1)}\left(r_{1} \bar{y}_{1}^{(1, \mu)}\right)^{j} .
$$

Finally, using the above relations and observing that $r_{j L}=L^{\alpha} r_{j}$, we conclude that

(i) for $i>j: \alpha_{j L, i L}^{(n)}=-r_{i L}\left(r_{j L} \bar{y}_{j L}^{(L, n)}\right)=-r_{i} L^{\alpha} L^{-\alpha} \beta_{j}^{(1)}\left(r_{1} \bar{y}_{1}^{(1, \mu)}\right)^{j}=-r_{i} r_{j} \bar{y}_{j}^{(1, \mu)}=$ $\alpha_{j, i}^{(\mu)}$. 
(ii) for $i=j: \alpha_{j L, j L}^{(n)}=1-r_{j L} r_{L}^{-1}-r_{j L}^{2} \bar{y}_{j L}^{(L, n)}=1-r_{j} r_{1}^{-1}-r_{j} \beta_{j}^{(1)}\left(r_{1} \bar{y}_{1}^{(1, \mu)}\right)^{j}=$ $1-r_{j} r_{1}^{-1}-r_{j}^{2} \bar{y}_{j}^{(1, \mu)}=\alpha_{j, j}^{(\mu)}$.

(iii) for $i<j$, using the computations done in case (i) we have $\alpha_{j L, i L}^{(n)}=-r_{i L} r_{j L} \bar{y}_{j L}^{(L, n)}+$ $r_{i L} r_{(j-i) L} \bar{y}_{(j-i) L}^{(L, n)}=-r_{i} r_{j} \bar{y}_{j}^{(1, \mu)}+r_{j-i} \bar{y}_{j-i}^{(1, \mu)}=\alpha_{j, i}^{(\mu)}$.

Hence, $\mathcal{K}^{(L, n)}=\mathcal{K}^{(1, \mu)}$, as we wanted to prove.

Remark 4. If Conjecture 1 is true, then Proposition 13 holds without the restriction on $\mu$ and this, together with the invariance properties of the sets $\mathcal{S}_{M}$, implies that the unstable directions of $\bar{y}^{(L, n)}$ in $\overline{\mathcal{S}_{M}}$ have a nonzero component in $\overline{\mathcal{S}_{M}}-\mathcal{S}_{L}$. This observation will be important for the analysis of the numerical results in Section 4.3.

\subsection{Linear Stability Results in the Case $r_{j}=j$}

In this section we prove Conjecture 1 when $r_{j}=j$. The proof is particular for this case and is not likely to be applicable to more general sequences. Nevertheless, we include it here for three reasons: because it is the only case for which Conjecture 1 has been proved so far; because these coefficients are actually important in gelation studies (cf. (P4) in the introduction); and finally, because it proves that the surprising behaviour of the spectra of $\mathcal{K}^{(1, \mu)}$ shown in Table 3 is indeed true. The main result is the following.

Proposition 15. Let $r_{j}=j$, and let $\mathcal{K}^{(1, \mu)}$ be the matrix defined by (19) with $\mu \geq 2$. Then,

$$
\sigma_{\mathcal{K}}=\{\lambda=-\Lambda: \Lambda=1, \ldots, \mu-1\} \bigcup\left\{-\sum_{j=1}^{\mu} \frac{j^{j}}{j !} \bar{y}^{j}\right\}
$$

where $\bar{y}:=\bar{y}_{1}^{(1, \mu)}$.

Proof. We start by noting that $r_{j}=j$ implies $\beta_{j}^{(1)}=\frac{j^{j-1}}{j !}$. Thus, the elements $\alpha_{j, i}:=\alpha_{j, i}^{(\mu)}$ of $\mathcal{K}^{(1, \mu)}$ can be written as

$$
\alpha_{j, i}= \begin{cases}-i \frac{j^{j-1}}{j !} \bar{y}^{j}, & \text { if } i>j, \\ 1-j-\frac{j^{j}}{j !} \bar{y}^{j}, & \text { if } i=j, \\ -i \frac{j^{j-1}}{j !} \bar{y}^{j}+i \frac{(j-i)^{j-i-1}}{(j-i) !} \bar{y}^{j-i}, & \text { if } i<j .\end{cases}
$$

Since the trace of $\mathcal{K}^{(1, \mu)}$ is easily computed, the eigenvalue of $\mathcal{K}^{(1, \mu)}$ different from $-\Lambda$ is easily obtained: Assuming that $-1, \ldots,-(\mu-1)$ are eigenvalues of $\mathcal{K}^{(1, \mu)}$, then 
the remaining eigenvalue is

$$
\begin{aligned}
\lambda & =\operatorname{tr} \mathcal{K}^{(1, \mu)}-\sum_{j=1}^{\mu-1}(-j) \\
& =\sum_{j=1}^{\mu}\left(1-j-\frac{j^{j}}{j !} \bar{y}^{j}\right)+\sum_{j=1}^{\mu-1} j \\
& =\mu-\sum_{j=1}^{\mu} j-\sum_{j=1}^{\mu} \frac{j^{j}}{j !} \bar{y}^{j}+\sum_{j=1}^{\mu-1} j \\
& =-\sum_{j=1}^{\mu} \frac{j^{j}}{j !} \bar{y}^{j} .
\end{aligned}
$$

We are now left to prove that $-\Lambda$ with $\Lambda=1, \ldots, \mu-1$ are eigenvalues of $\mathcal{K}^{(1, \mu)}$. This will be done by showing that, for each of these values, the matrices $\mathcal{K}^{(1, \mu)}+\Lambda I_{\mu}$ are singular; more specifically, it will be shown that the $(\Lambda+1)^{\text {th }}$ line of these matrices can be written as a linear combination of the previous $\Lambda$ lines. In order not to overload the notation, let $\left[j^{\text {th }}\right]$ denote the $j^{\text {th }}$ line of $\mathcal{K}^{(1, \mu)}+\Lambda I_{\mu}$, let [new $1^{\text {st }}$ be the line obtained by multiplication of the first line of $\mathcal{K}^{(1, \mu)}+\Lambda I_{\mu}$ by $\frac{(\Lambda+1)^{\Lambda}}{(\Lambda+1) !} \bar{y}^{\Lambda}$, that is,

$$
\left[\text { new } 1^{\text {st }}\right]=\frac{(\Lambda+1)^{\Lambda}}{(\Lambda+1) !} \bar{y}^{\Lambda}\left[1^{\text {st }}\right]
$$

and, for $j \geq 2$, let

$$
\left[\text { new } j^{\text {th }}\right]=\bar{y}^{\Lambda-j+1}\left[j^{\text {th }}\right]-\frac{j^{j-1}}{j !} \bar{y}^{\Lambda}\left[1^{\mathrm{st}}\right]
$$

We shall prove that

$$
\left[(\Lambda+1)^{\mathrm{th}}\right]=\left[\text { new } 1^{\mathrm{st}}\right]+\sum_{j=2}^{\Lambda}(-1)^{\Lambda-j} \frac{j^{\Lambda-j+1}}{(\Lambda-j+1) !}\left[\text { new } j^{\text {th }}\right]
$$

where the sum is defined to be zero if $\Lambda=1$.

Let us start by the case $\Lambda=1$. We are going to prove that $\left[2^{\text {nd }}\right]=\left[\right.$ new $\left.1^{\text {st }}\right]$. Considering first what happens with components $i>\Lambda=1$, we have that the $i^{\text {th }}$ component of [new $1^{\text {st }}$ ] is

$$
\left[\left(\text { new } 1^{\mathrm{st}}\right)_{i}\right]=\frac{2^{1}}{2 !} \bar{y} \alpha_{1, i}=-\frac{2^{1}}{2 !} \bar{y} i \frac{1^{0}}{1 !} \bar{y}=-i \bar{y}^{2},
$$

and, on the other hand, $\left[\left(2^{\text {nd }}\right)_{i}\right]$ is given by

$$
\alpha_{2, i}+\delta_{2, i}=\left\{\begin{array}{ll}
-1-2 \bar{y}^{2}+1 & \text { if } i=2 \\
-i \frac{2^{1}}{2 !} \bar{y}^{2} & \text { if } i>2
\end{array}=\left\{\begin{array}{ll}
-2 \bar{y}^{2} & \text { if } i=2 \\
-i \bar{y}^{2} & \text { if } i>2
\end{array}=-i \bar{y}^{2}\right.\right.
$$


and so both are equal. For the first component, $i=1$, we have

$$
\left[\left(\text { new } 1^{\text {st }}\right)_{1}\right]=\frac{2^{1}}{2 !} \bar{y}\left(\alpha_{1,1}+\delta_{1,1}\right)=\bar{y}-\bar{y}^{2}=\alpha_{2,1}=\left[\left(2^{\text {nd }}\right)_{1}\right] .
$$

This concludes the proof that -1 is an eigenvalue of $\mathcal{K}^{(1, \mu)}$.

We now consider a general integer $\Lambda$ satisfying $2 \leq \Lambda \leq \mu-1$ and will show that (26) holds. We start with components $i>\Lambda$. The sum in the right-hand side of (26) is equal to

$$
\begin{aligned}
& \sum_{j=2}^{\Lambda}(-1)^{\Lambda-j} \frac{j^{\Lambda-j+1}}{(\Lambda-j+1) !}\left(\bar{y}^{\Lambda-j+1}\left(\alpha_{j, i}+\Lambda \delta_{j, i}\right)-\frac{j^{j-1}}{j !} \bar{y}^{\Lambda}\left(\alpha_{1, i}+\Lambda \delta_{1, i}\right)\right) \\
& \quad=\sum_{j=2}^{\Lambda}(-1)^{\Lambda-j} \frac{j^{\Lambda-j+1}}{(\Lambda-j+1) !}\left(\bar{y}^{\Lambda-j+1}(-i) \frac{j^{j-1}}{j !} \bar{y}^{j}-\frac{j^{j-1}}{j !} \bar{y}^{\Lambda}(-i) 1 \bar{y}\right) \\
& \quad=0,
\end{aligned}
$$

and $\left[\left(\text { new } 1^{\text {st }}\right)_{i}\right]=-i \frac{(\Lambda+1)^{\Lambda}}{(\Lambda+1) !} \bar{y}^{\Lambda+1}$. For the left-hand side of (26), we have

$$
\begin{aligned}
{\left[\left((\Lambda+1)^{\mathrm{th}}\right)_{i}\right] } & = \begin{cases}1-(\Lambda+1)-\frac{(\Lambda+1)^{\Lambda+1}}{(\Lambda+1) !} \bar{y}^{\Lambda+1}+\Lambda & \text { if } i=\Lambda+1 \\
-i \frac{(\Lambda+1)^{\Lambda}}{(\Lambda+1) !} \bar{y}^{\Lambda+1} & \text { if } i>\Lambda+1\end{cases} \\
& = \begin{cases}-\frac{(\Lambda+1)^{\Lambda+1}}{(\Lambda+1) !} \bar{y}^{\Lambda+1} & \text { if } i=\Lambda+1 \\
-i \frac{(\Lambda+1)^{\Lambda}}{(\Lambda+1) !} \bar{y}^{\Lambda+1} & \text { if } i>\Lambda+1 \\
& =-i \frac{(\Lambda+1)^{\Lambda}}{(\Lambda+1) !} \bar{y}^{\Lambda+1},\end{cases}
\end{aligned}
$$

and so (26) holds for components $i>\Lambda$.

If $i=\Lambda$, we have, for the sum in the right-hand side of (26),

$$
\begin{aligned}
& \sum_{j=2}^{\Lambda}(-1)^{\Lambda-j} \frac{j^{\Lambda-j+1}}{(\Lambda-j+1) !}\left(\bar{y}^{\Lambda-j+1}\left(\alpha_{j, \Lambda}+\Lambda \delta_{j, \Lambda}\right)-\frac{j^{j-1}}{j !} \bar{y}^{\Lambda}\left(\alpha_{1, \Lambda}+\Lambda \delta_{1, \Lambda}\right)\right) \\
& =\sum_{j=2}^{\Lambda-1}(-1)^{\Lambda-j} \frac{j^{\Lambda-j+1}}{(\Lambda-j+1) !}\left(\bar{y}^{\Lambda-j+1}(-\Lambda) \frac{j^{j-1}}{j !} \bar{y}^{j}-\frac{j^{j-1}}{j !} \bar{y}^{\Lambda}(-\Lambda) 1 \bar{y}\right) \\
& \quad+(-1)^{\Lambda-\Lambda} \frac{\Lambda^{\Lambda-\Lambda+1}}{(\Lambda-\Lambda+1) !}\left(\bar{y}^{\Lambda-\Lambda+1}\left(1-\Lambda-\frac{\Lambda^{\Lambda}}{\Lambda !} \bar{y}^{\Lambda}+\Lambda\right)+\frac{\Lambda^{\Lambda}}{\Lambda !} \bar{y}^{\Lambda+1}\right) \\
& =0+\Lambda \bar{y} \\
& =\Lambda \bar{y},
\end{aligned}
$$


and

$$
\begin{aligned}
& {\left[\left((\Lambda+1)^{\mathrm{th}}\right)_{\Lambda}\right]-\left[\left(\text { new } 1^{\mathrm{st}}\right)_{\Lambda}\right]} \\
& \quad=\left(-\Lambda \frac{(\Lambda+1)^{\Lambda}}{(\Lambda+1) !} \bar{y}^{\Lambda+1}+\Lambda \bar{y}\right)-\left(-\frac{(\Lambda+1)^{\Lambda}}{(\Lambda+1) !} \bar{y}^{\Lambda}(-\Lambda) \bar{y}\right) \\
& =\Lambda \bar{y},
\end{aligned}
$$

and thus (26) holds for component $i=\Lambda$.

Consider now $1<i<\Lambda$. The sum in the right-hand side of (26) becomes

$$
\begin{aligned}
\sum_{j=2}^{\Lambda}(-1)^{\Lambda-j} & \frac{j^{\Lambda-j+1}}{(\Lambda-j+1) !}\left(\bar{y}^{\Lambda-j+1}\left(\alpha_{j, i}+\Lambda \delta_{j, i}\right)-\frac{j^{j-1}}{j !} \bar{y}^{\Lambda}\left(\alpha_{1, i}+\Lambda \delta_{1, i}\right)\right) \\
= & \sum_{j=2}^{i-1}(-1)^{\Lambda-j} \frac{j^{\Lambda-j+1}}{(\Lambda-j+1) !}\left(\bar{y}^{\Lambda+1}(-i) \frac{j^{j-1}}{j !}-\frac{j^{j-1}}{j !} \bar{y}^{\Lambda}(-i) 1 \bar{y}\right) \\
& +(-1)^{\Lambda-i} \frac{i^{\Lambda-i+1}}{(\Lambda-i+1) !}\left(\bar{y}^{\Lambda-i+1}\left(1-i-\frac{i^{i}}{i !} \bar{y}^{i}+\Lambda\right)+\frac{i^{i}}{i !} \bar{y}^{\Lambda+1}\right) \\
& +\sum_{j=i+1}^{\Lambda}(-1)^{\Lambda-j} \frac{j^{\Lambda-j+1}}{(\Lambda-j+1) !}\left(\bar{y}^{\Lambda-j+1}\left(-i \frac{j^{j-1}}{j !} \bar{y}^{j}+i \frac{(j-i)^{j-i+1}}{(j-i) !} \bar{y}^{j-i}\right)\right. \\
= & 0+(-1)^{\Lambda-i} \frac{i^{\Lambda-i+1}}{(\Lambda-i+1) !} \bar{y}^{\Lambda-i+1}(\Lambda-i+1) \\
& +i \bar{y}^{\Lambda-i+1} \sum_{j=i+1}^{\Lambda}(-1)^{\Lambda-j} \frac{j^{\Lambda-j+1}}{(\Lambda-j+1) !} \frac{(j-i)^{j-i+1}}{(j-i) !} .
\end{aligned}
$$

And, as we have

$$
\begin{aligned}
& {\left[\left((\Lambda+1)^{\mathrm{th}}\right)_{i}\right]-\left[\left(\text { new } 1^{\mathrm{st}}\right)_{i}\right]} \\
& \quad=\left(-i \frac{(\Lambda+1)^{\Lambda}}{(\Lambda+1) !} \bar{y}^{\Lambda+1}+i \frac{(\Lambda-i+1)^{\Lambda-i}}{(\Lambda-i+1) !} \bar{y}^{\Lambda-i+1}\right)-\left(\frac{(\Lambda+1)^{\Lambda}}{(\Lambda+1) !} \bar{y}^{\Lambda}(-i) 1 \bar{y}\right) \\
& \quad=i \frac{(\Lambda-i+1)^{\Lambda-i}}{(\Lambda-i+1) !} \bar{y}^{\Lambda-i+1},
\end{aligned}
$$

we conclude that (26) becomes

$$
\sum_{j=i+1}^{\Lambda}(-1)^{\Lambda-j} \frac{j^{\Lambda-j+1}}{(\Lambda-j+1) !} \frac{(j-i)^{j-i+1}}{(j-i) !}=\frac{(\Lambda-i+1)^{\Lambda-i}}{(\Lambda-i+1) !}-(-1)^{\Lambda-i} \frac{i^{\Lambda-i}}{(\Lambda-i) !} .
$$

Observing that $\frac{(\Lambda-i+1)^{\Lambda-i}}{(\Lambda-i+1) !}$ is the term $j=\Lambda+1$ in the sum of the left-hand side, we can write the expression above as

$$
\sum_{j=i+1}^{\Lambda+1}(-1)^{\Lambda-j+1} \frac{j^{\Lambda-j+1}}{(\Lambda-j+1) !} \frac{(j-i)^{j-i+1}}{(j-i) !}=(-1)^{\Lambda-i} \frac{i^{\Lambda-i}}{(\Lambda-i) !} .
$$


Now change the summation variable from $j$ to $j-i$. Still denoting the new variable by $j$ and defining $\tilde{\Lambda}:=\Lambda-i+1$, the last expression becomes

$$
\frac{1}{\tilde{\Lambda} !} \sum_{j=1}^{\tilde{\Lambda}}(-1)^{\tilde{\Lambda}-j}\left(\begin{array}{c}
\tilde{\Lambda} \\
j
\end{array}\right)(j+i)^{\tilde{\Lambda}-j} j^{j-1}=(-1)^{\tilde{\Lambda}-1} \frac{i^{\tilde{\Lambda}-1}}{(\tilde{\Lambda}-1) !} .
$$

To prove (27) we will basically expand $(j+i)^{\tilde{\Lambda}-j}$, rearrange the terms, and change the order of summation:

$$
\begin{aligned}
\frac{1}{\tilde{\Lambda} !} \sum_{j=1}^{\tilde{\Lambda}}(-1)^{\tilde{\Lambda}-j}\left(\begin{array}{c}
\tilde{\Lambda} \\
j
\end{array}\right)(j+i)^{\tilde{\Lambda}-j} j^{j-1} \\
=\frac{1}{\tilde{\Lambda} !} \sum_{j=1}^{\tilde{\Lambda}}(-1)^{\tilde{\Lambda}-j}\left(\begin{array}{c}
\tilde{\Lambda} \\
j
\end{array}\right) \sum_{p=0}^{\tilde{\Lambda}-j}\left(\begin{array}{c}
\tilde{\Lambda}-j \\
p
\end{array}\right) j^{\tilde{\Lambda}-j-p} i^{p} j^{j-1} \\
=\frac{1}{\tilde{\Lambda} !} \sum_{j=1}^{\tilde{\Lambda}} \sum_{p=0}^{\tilde{\Lambda}-j}(-1)^{\tilde{\Lambda}-j}\left(\begin{array}{c}
\tilde{\Lambda} \\
j
\end{array}\right)\left(\begin{array}{c}
\tilde{\Lambda}-j \\
p
\end{array}\right) j^{\tilde{\Lambda}-p-1} i^{p} \\
=\frac{1}{\tilde{\Lambda} !} \sum_{j=1}^{\tilde{\Lambda}} \sum_{p=0}^{\tilde{\Lambda}-j}(-1)^{\tilde{\Lambda}-j}\left(\begin{array}{c}
\tilde{\Lambda} \\
p
\end{array}\right)\left(\begin{array}{c}
\tilde{\Lambda}-p \\
j
\end{array}\right) j^{\tilde{\Lambda}-p-1} i^{p} \\
=\frac{1}{\tilde{\Lambda} !} \sum_{p=0}^{\tilde{\Lambda}-1}(-1)^{p}\left(\begin{array}{c}
\tilde{\Lambda} \\
p
\end{array}\right) i^{p} \sum_{j=1}^{\tilde{\Lambda}-p}(-1)^{(\tilde{\Lambda}-p)-j}\left(\begin{array}{c}
\tilde{\Lambda}-p \\
j
\end{array}\right) j^{\tilde{\Lambda}-p-1} \\
=\frac{1}{\tilde{\Lambda} !} \sum_{p=0}^{\tilde{\Lambda}-2}(-1)^{p}\left(\begin{array}{c}
\tilde{\Lambda} \\
p
\end{array}\right) i^{p} S(\tilde{\Lambda}-p-1, \tilde{\Lambda}-p)(\tilde{\Lambda}-p) ! \\
\quad+\frac{1}{\tilde{\Lambda} !}(-1)^{\tilde{\Lambda}-1}\left(\begin{array}{c}
\tilde{\Lambda} \\
\tilde{\Lambda}-1
\end{array}\right) i^{\tilde{\Lambda}-1} \\
=(-1)^{\tilde{\Lambda}-1} \frac{i^{\tilde{\Lambda}-1}}{(\tilde{\Lambda}-1) !},
\end{aligned}
$$

where $S(n, k)$ is the Stirling partition number, or Stirling number of the second kind [22], which is

$$
S(n, k)=\frac{1}{k !} \sum_{j=0}^{k}(-1)^{k-j}\left(\begin{array}{l}
k \\
j
\end{array}\right) j^{n},
$$

and the last equality in the deduction above is due to the fact that $S(n, k)=0$ if $n<k$ [22, p. 163]. This concludes the proof for the lines $1<i<\Lambda$.

The final case $i=1$ is similar to the previous one, and we shall skip most of the details: The sum in the right-hand side of (26) can be written in the form

$$
\sum_{j=2}^{\Lambda}(-1)^{\Lambda-j} \frac{j^{\Lambda-j+1}}{(\Lambda-j+1) !}\left(\frac{(j-1)^{j-2}}{(j-1) !}-\Lambda \frac{j^{j-1}}{j !}\right) \bar{y}^{\Lambda},
$$


and

$$
\left[\left((\Lambda+1)^{\mathrm{th}}\right)_{i}\right]-\left[\left(\text { new } 1^{\mathrm{st}}\right)_{i}\right]=\left(\frac{\Lambda^{\Lambda-1}}{\Lambda !}-\Lambda \frac{(\Lambda+1)^{\Lambda}}{(\Lambda+1) !}\right) \bar{y}^{\Lambda}
$$

which is equal to the symmetric of the term of the sum corresponding to $j=\Lambda+1$. This means that, after some minor rearrangements, we can write (26) in the form

$$
\frac{1}{\Lambda !} \sum_{j=1}^{\Lambda}(-1)^{\Lambda-j}\left(\begin{array}{l}
\Lambda \\
j
\end{array}\right)(j+1)^{\Lambda-j}\left(\Lambda(j+1)^{j-1}-j^{j-1}\right)=0 .
$$

We start by looking at

$$
\frac{\Lambda}{\Lambda !} \sum_{j=1}^{\Lambda}(-1)^{\Lambda-j}\left(\begin{array}{l}
\Lambda \\
j
\end{array}\right)(j+1)^{\Lambda-1},
$$

which is evaluated in exactly the same manner as for the left-hand side of (27) to give

$$
\begin{aligned}
& \frac{\Lambda}{\Lambda !} \sum_{p=1}^{\Lambda-1}(\Lambda-1) S(p, \Lambda) \Lambda !+\frac{\Lambda}{\Lambda !}(-1)^{\Lambda} \sum_{j=1}^{\Lambda}(-1)^{j}\left(\begin{array}{l}
\Lambda \\
j
\end{array}\right) \\
& \quad=0+\frac{\Lambda}{\Lambda !}(-1)^{\Lambda}\left(\sum_{j=0}^{\Lambda}(-1)^{j}\left(\begin{array}{l}
\Lambda \\
j
\end{array}\right)-(1)^{0}\left(\begin{array}{l}
\Lambda \\
0
\end{array}\right)\right) \\
& \quad=-\frac{(-1)^{\Lambda}}{(\Lambda-1) !} .
\end{aligned}
$$

The other term in (28), namely,

$$
\frac{1}{\Lambda !} \sum_{j=1}^{\Lambda}(-1)^{\Lambda-j}\left(\begin{array}{c}
\Lambda \\
j
\end{array}\right) j^{j-1}(j+1)^{\Lambda-j},
$$

is equal to the left-hand side of (27) with $i=1$ and hence it is also equal to $-\frac{(-1)^{\Lambda}}{(\Lambda-1) !}$. This proves (26) for the component $i=1$ and concludes the proof of the proposition.

The results of Propositions 14 and 15 imply that Proposition 13 holds true without restrictions on $\mu$.

\subsection{Global Behaviour of Nonnegative Solutions}

A problem that naturally arises after the study of the dynamics near the equilibria, done in Sections 4.1 and 4.2, is the elucidation of the global behaviour.

Based on a large body of numerical evidence, a small part of which will be presented below, we state the following conjecture.

Conjecture 2. Assume (H1). For all $L \in\{1,2, \ldots, n\}$, the equilibria $\bar{y}^{(L, n)}$ are globally asymptotically stable in $\mathcal{S}_{L}$. 
We could only prove global stability for the cases $L=n-1$ and $L=n$, and this is made using methods not applicable to other values of $L$.

Proposition 16. The equilibria $\bar{y}^{(L, n)}$ are globally asymptotically stable in $\mathcal{S}_{L}$ for $L=$ $n-1$ and $L=n$.

Proof. Both cases are extremely simple. For $L=n$, the set $\overline{\mathcal{S}_{L}}$ can be identified with $\mathbb{R}_{0}^{(n-L+1)+}=\mathbb{R}_{0}^{+}$and so the restriction of (10) to $\overline{\mathcal{S}_{n}}$ is one-dimensional, namely, $y_{n}^{\prime}=$ $F_{n}\left(y_{n}\right)$ with $F_{n}$ as given in the proof of Proposition 7, and this equation has $y_{n}=r_{n}^{-2}$ as the global attractor for positive solutions. Consequently, $\bar{y}^{(L, n)}=\left(0, \ldots, 0, r_{n}^{-2}\right)$ is the global attractor of (10) restricted to $\mathcal{S}_{n}$. For $L=n-1$, the set $\overline{\mathcal{S}_{L}}$ can be identified with $\mathbb{R}_{0}^{2+}$, and the restriction of (10) to this set is, for $n>2$,

$$
\left\{\begin{array}{l}
y_{n-1}^{\prime}=y_{n-1}-r_{n-1} y_{n-1}\left(r_{n-1} y_{n-1}+r_{n} y_{n}\right), \\
y_{n}^{\prime}=y_{n}-r_{n} y_{n}\left(r_{n-1} y_{n-1}+r_{n} y_{n}\right)
\end{array}\right.
$$

Since, by Proposition 7, nonnegative solutions of (10) are bounded, we conclude that nonnegative solutions to (29) are also bounded. Since the only equilibria of (29) are $(0,0), \bar{y}^{(n-1, n)}=\left(r_{n-1}^{-2}, 0\right)$, and $\bar{y}^{(n, n)}=\left(0, r_{n}^{-2}\right)$, the local stability results of Section 4.1 and the Poincaré-Bendixon theorem allow us to conclude that $\bar{y}^{(n-1, n)}$ is the global attractor of (10) restricted to $\mathcal{S}_{n-1}$.

It is clear that the proof above is not applicable to higher-dimensional systems, i.e., to smaller values of $L$.

We next present some of the numerical evidence for Conjecture 2. The overall qualitative picture that one obtains is independent of the coefficients $\left(r_{j}\right)$ provided they satisfy (H1). Hence, we will present results only for the rate coefficients $r_{j}=j$ since for this case the local behaviour near equilibria was already established in Propositions 11, 14, and 15 . For other cases, the interpretation of the observed behaviour near equilibria must rest on the assumption of the validity of Conjecture 1.

In interpreting the numerical results, a few facts should be remembered:

(i) The nonzero components $y_{j}(\tau)$ of the solution $y(\tau)$ are those given by Proposition 2 .

(ii) The only nonnegative equilibria of (10) are those given by Proposition 8 , and, in particular, the nonzero components of any equilibrium $\bar{y}^{(L, n)}$ are those for which the subscripts $j$ are multiples of $L$, as was already pointed out in Remark 2.

(iii) The behaviour of solutions near equilibria is governed by the results in Propositions 11, 14, and 15, which imply that Proposition 13 holds without restrictions upon $\mu$, and so also does Remark 4 about the unstable directions of the equilibria.

The graphics presented are those of numerically computed $y_{j}(\tau)$ as a function of $\tau$ for the several values of $j \in\{1, \ldots, n\}$ under different types of initial data and truncation size $n$. In each graphic, the component $j$ is indicated by the corresponding number. Before proceeding to the numerical results, just a brief note about notation: The initial data for (10) is a vector $y_{0} \in \mathbb{R}_{0}^{n+}$. In presenting the initial data used in each experiment, we shall give the list of the components of $y_{0}$ in the following way: Instead of writing $y_{0}=\left(\alpha_{1}, \alpha_{2}, \ldots, \alpha_{n}\right)$, we shall write $y_{0 j}=\sum_{i=1}^{n} \alpha_{i} \delta_{j, i}$. 
We start, in Figure 3, with some simple cases illustrating that solutions with initial data in $\mathcal{S}_{L}$ converge to $\bar{y}^{(L)}$ as $\tau \rightarrow+\infty$.

In the cases (a) and (b) of Figure 3, the initial data is monodispersed (i.e., only one component of $y_{0}$ is nonzero), and in both cases numerical solutions converge to the expected $\bar{y}^{(L)}$. Case (c) is a typical result with "small" arbitrary initial data.

In the next set of experiments, shown in Figure 4, we present situations where the initial data $y_{0}$ is very close to an equilibrium: Namely, we use $n=21$ and initial data very close to $\bar{y}^{(21)}$.

We again observe that $y_{0} \in \mathcal{S}_{L}$ implies that $y(\tau) \longrightarrow \bar{y}^{(L)}$ as $\tau \rightarrow+\infty$. In all three cases (a), (b), and (c) we take initial data that is a perturbation of the equilibrium $\bar{y}^{(21)}$ by the addition of $10^{-7}$ to the component $L=6,5$, and 3, respectively. We clearly observe that the solutions follow heteroclinic orbits connecting $\bar{y}^{(21)}$ to $\bar{y}^{(L)}$.

The last set of experiments is intended to pursue this type of observation a step further: In Figure 5 we show that by carefully choosing the initial data in $\mathcal{S}_{L}$ near an equilibrium in $\partial \mathcal{S}_{L}$, we can obtain a solution cascading down a number of equilibria before getting close to the limit equilibrium $\bar{y}^{(L)}$.

In both cases of Figure 5, the initial data is a small perturbation of the equilibrium $\bar{y}^{(21)}$ and lies in $\mathcal{S}_{2}$. Hence, according to Conjecture 2, it is expected that in both cases the solutions converge to $\bar{y}^{(2)}$, as is in fact the case (at least numerically). By carefully choosing the way we perturb $\bar{y}^{(21)}$, we can force the solution to follow a given network of heteroclinic orbits. In case (a), the initial data is a small perturbation of $\bar{y}^{(21)}$ with a dominant fourth component, since $1 \gg y_{04} \gg y_{02}$. Thus, the system behaves in the short time as if $y_{0} \in \mathcal{S}_{4}$, and the solution follows a heteroclinic orbit from $\bar{y}^{(21)}$ to $\bar{y}^{(4)}$. After some time near $\bar{y}^{(4)}$, the solution finally converges to the limit equilibrium $\bar{y}^{(2)}$. In case (b), the transient behaviour is modified by now choosing an initial data that is a small perturbation of $\bar{y}^{(21)}$ with $1 \gg y_{09} \gg y_{05} \gg y_{03} \gg y_{02}$. If this is done carefully, we can capture numerically a solution that follows the network of heteroclinic orbits connecting $\bar{y}^{(21)}$ to $\bar{y}^{(9)}$ to $\bar{y}^{(5)}$ to $\bar{y}^{(3)}$ and, finally, to $\bar{y}^{(2)}$, as shown in (b).

These results and the robustness of the numerical behaviour seem to indicate not only that Conjecture 2 is true, but also that (10) is in fact a Morse-Smale system.

\section{Final Remarks}

Of the two conjectures made in this paper, Conjecture 2 on the global dynamics of (10), is clearly the most important. Its proof will, likely, entail the discovery of appropriate Lyapunov functionals $V_{n}$ for the $n$-dimensional system (10), for all $n \in \mathbb{N}$. If the Lyapunov functionals are constructed in a convenient manner, it should be possible to prove that, as $n \rightarrow \infty$, the functionals $V_{n}$ would converge, in an appropriate sense, to some functional $V$, at least for some types of rate coefficients $a_{j, k}$. By showing the limit functional $V$ to be a Lyapunov functional for the limit system obtained from (10) by letting $n \rightarrow \infty$, one would probably be able to prove rigorously the occurrence of gelation for extended families of rate coefficients and initial data.

This program obviously is also connected to the one of relating the attractors of each of the finite $n$-dimensional systems (10) with the attractor of the infinite-dimensional limit system, which has an infinite number of equilibria, $\alpha^{(L)}$, componentwise convergent to zero as $L \rightarrow \infty$.

We hope to return to these problems in the future. 


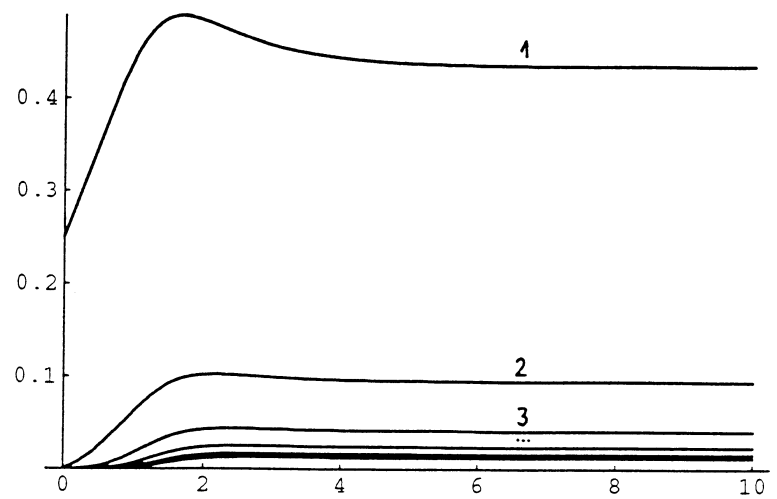

(a)

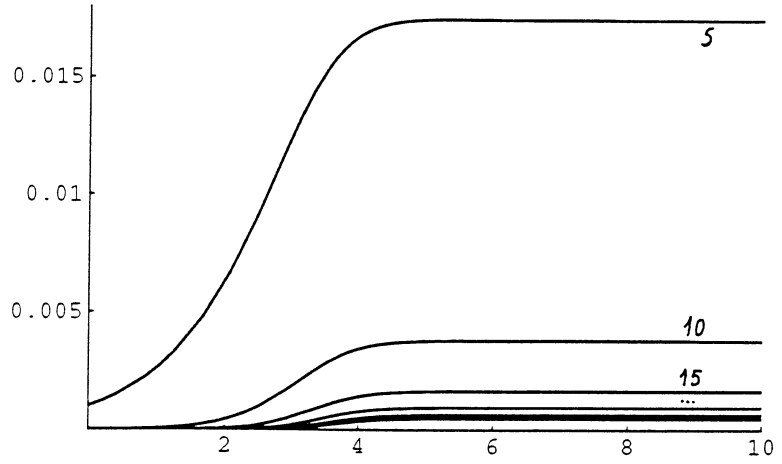

(b)

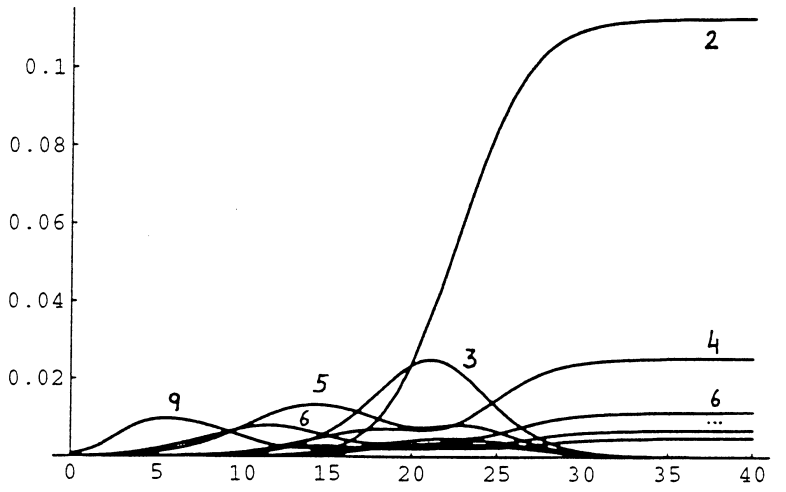

(c)

Fig. 3. Numerical solutions to (10) for $a_{j, k}=j k$ with (a) $n=6$, $y_{0 j}=0.25 \delta_{j, 1}$; (b) $n=30, y_{0 j}=10^{-3} \delta_{j, 5}$; (c) $n=10, y_{0 j}=$ $2 \cdot 10^{-8} \delta_{j, 2}+4 \cdot 10^{-7} \delta_{j, 3}+2 \cdot 10^{-6} \delta_{j, 4}+5 \cdot 10^{-5} \delta_{j, 5}+7 \cdot 10^{-5} \delta_{j, 6}+$ $8 \cdot 10^{-9} \delta_{j, 7}+7 \cdot 10^{-4} \delta_{j, 9}+2 \cdot 10^{-7} \delta_{j, 10}$. 


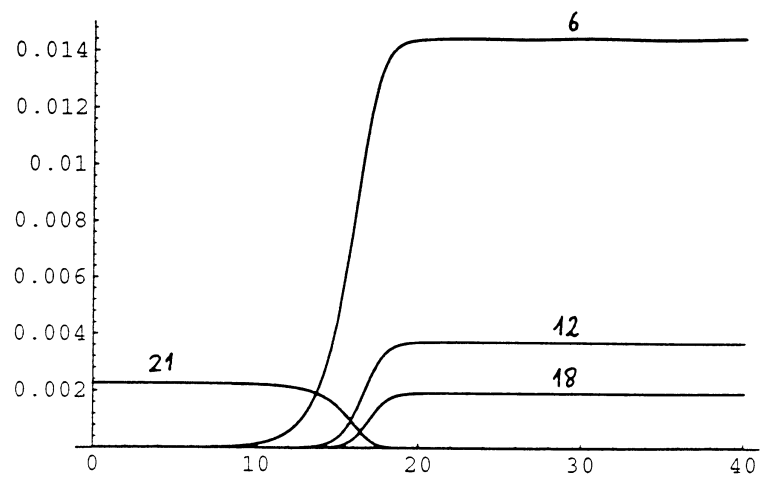

(a)

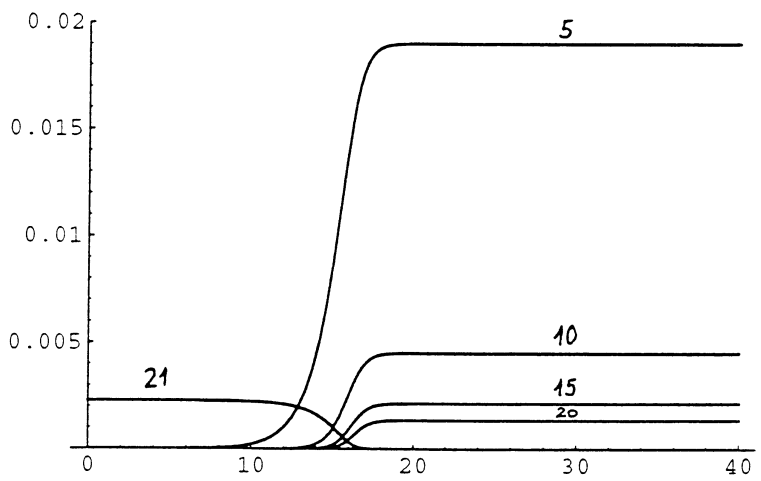

(b)

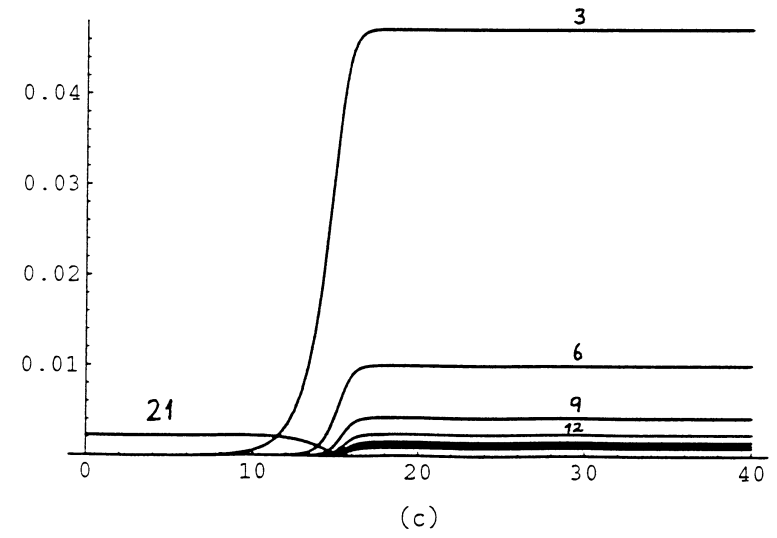

Fig. 4. Numerical solutions to (10) for $a_{j, k}=j k$ with $n=21$ and (a) $y_{0 j}=10^{-7} \delta_{j, 6}+0.00226757 \delta_{j, 21}$; (b) $y_{0 j}=10^{-7} \delta_{j, 5}+$ $0.00226757 \delta_{j, 21}$; (c) $y_{0 j}=10^{-7} \delta_{j, 3}+0.00226757 \delta_{j, 21}$. 


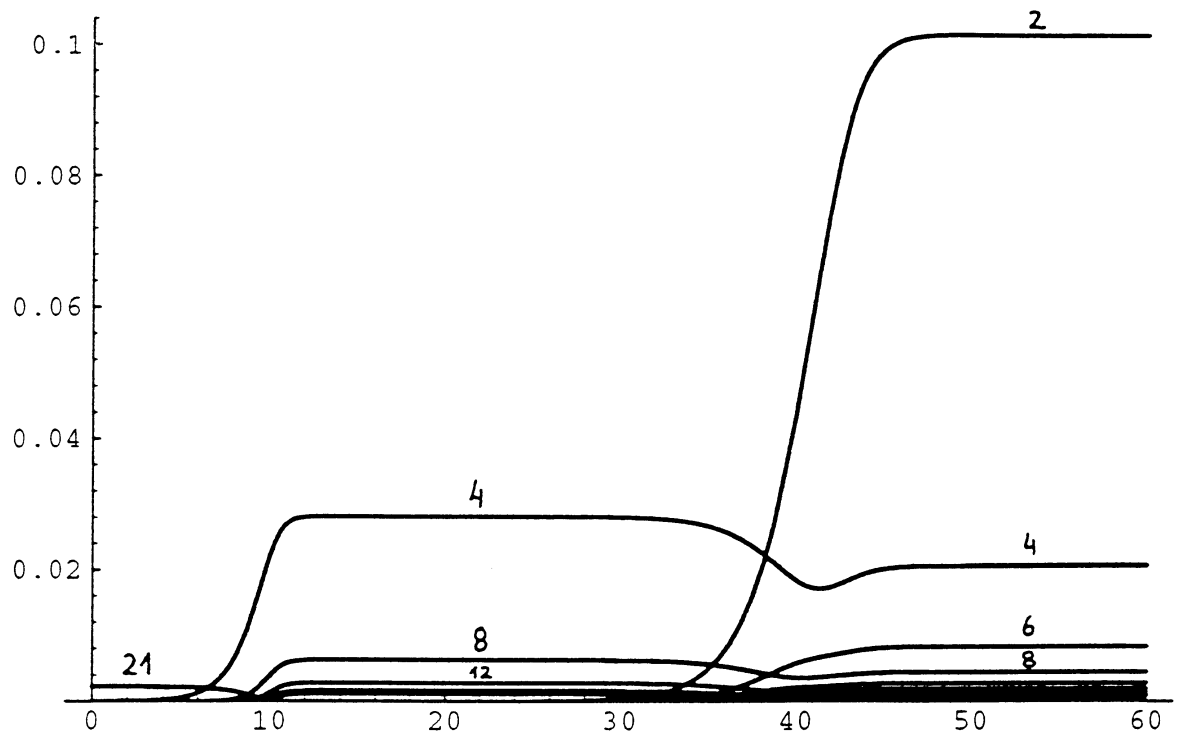

(a)

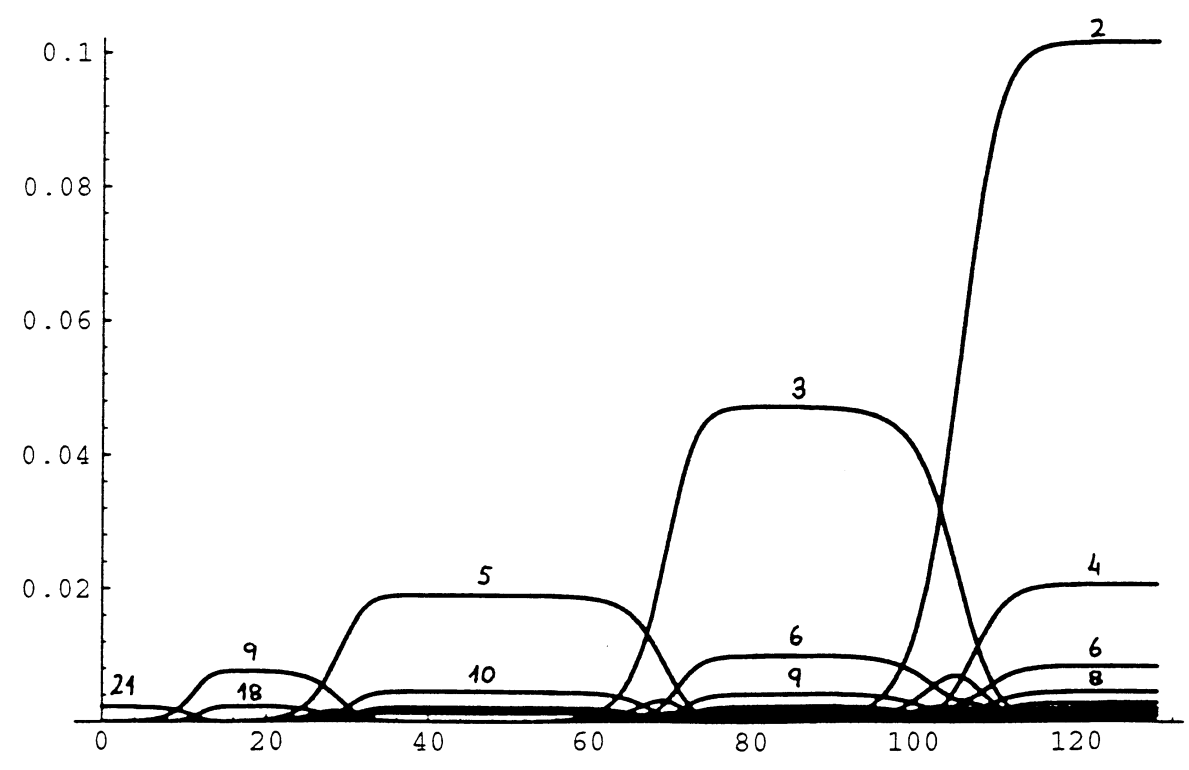

(b)

Fig. 5. Numerical solutions to (10) for $a_{j, k}=j k$ with $n=21$ and (a) $y_{0 j}=10^{-12} \delta_{j, 2}+10^{-5} \delta_{j, 4}+$ $0.00226757 \delta_{j, 21}$; (b) $y_{0 j}=10^{-28} \delta_{j, 2}+10^{-18} \delta_{j, 3}+10^{-9} \delta_{j, 5}+10^{-5} \delta_{j, 9}+0.00226757 \delta_{j, 21}$. 


\section{Acknowledgments}

I am grateful to Jack Carr, Pedro Freitas, Gero Friesecke, and Oliver Penrose for very stimulating discussions. This work was partially supported by FCT (Portugal) under projects PBIC/C/MAT/2139/95 and PRAXIS XXI Project 2/2.1/MAT/199/94.

\section{References}

[1] D. Adolf, J.E. Martin, The sol-gel transition in chemical gels, Ann. Rev. Phys. Chem., 42, 311-339 (1991).

[2] T.A. Bak, O. Heilmann, A finite version of Smoluchowski's coagulation equation, J. Phys. A: Math. Gen., 24, 4889-4893 (1991).

[3] J.M. Ball, J. Carr, The discrete coagulation-fragmentation equations: Existence, uniqueness, and density conservation, J. Stat. Phys., 61, 203-234 (1990).

[4] J.M. Ball, J. Carr, O. Penrose, The Becker-Döring cluster equations: Basic properties and asymptotic behaviour of solutions, Commun. Math. Phys., 104, 657-692 (1986).

[5] G. Benedek, R. Cohen, Equilibrium and kinetic theory of polymerization and the sol-gel transition, J. Phys. Chem., 86, 3696-3714 (1982).

[6] E. Buffet, J. Pulé, Gelation: The diagonal case revisited, Nonlinearity, 2, 373-381 (1989).

[7] J. Carr, F.P. da Costa, Instantaneous gelation in coagulation dynamics, Z. Angew. Math. Phys., 43, 974-983 (1992).

[8] J. Carr, F.P. da Costa, Asymptotic behavior of solutions to the coagulation-fragmentation equations. II. Weak fragmentation, J. Stat. Phys., 77, 89-123 (1994).

[9] S. Chandrasekhar, Stochastic problems in physics and astronomy, Rev. Mod. Phys., 15, 1-84 (1943).

[10] F.P. da Costa, Existence and uniqueness of density conserving solutions to the coagulationfragmentation equations with strong fragmentation, J. Math. Anal. \& Appl., 192, 892-914 (1995).

[11] F.P. da Costa, On the positivity of solutions to the Smoluchowski equations, Mathematika, 42, 406-412 (1995).

[12] R. Drake, A general mathematical survey of the coagulation equations, in J. Brock, G. Hidy, Topics in Current Aerosol Research (Part 2), International Reviews in Aerosol Physics and Chemistry, Pergamon Press, Oxford, 1972, pp. 201-376.

[13] M.H. Ernst, P. van Dongen, Cluster size distribution in irreversible aggregation at large times, J. Phys. A: Math. Gen., 18, 2779-2793 (1985).

[14] S. Friedlander, Smoke, Dust, and Haze, Wiley, New York, 1977.

[15] J. Heicklen, Colloid Formation and Growth, Academic Press, New York, 1976.

[16] E.M. Hendriks, M.H. Ernst, R.M. Ziff, Coagulation equations with gelation, J. Stat. Phys., 31, 519-563 (1983).

[17] P. Laurençot, Global solutions to the discrete coagulation equations, Les prépublications de l'Institut Élie Cartan, no. 28/97 (1997).

[18] F. Leyvraz, Existence and properties of post-gel solutions for the kinetic equations of coagulation, J. Phys. A: Math. Gen., 16, 2861-2873 (1983).

[19] F. Leyvraz, New exactly solvable models of Smoluchowski's equations of coagulation, $J$. Phys. A: Math. Gen., 18, 321-326 (1985).

[20] F. Leyvraz, H.R. Tschudi, Singularities in the kinetics of coagulation processes, J. Phys. A: Math. Gen., 14, 3389-3405 (1981).

[21] F. Leyvraz, H.R. Tschudi, Critical kinetics near gelation, J. Phys. A: Math. Gen., 15, 19511964 (1982).

[22] L. Lovász, Combinatorial Problems and Exercises, 2nd ed., North-Holland, Amsterdam, 1993.

[23] A.A. Lushnikov, V.N. Piskunov, Analytic solutions in the theory of coagulating systems with sinks, PMM USSR, 47, 743-750 (1980). 
[24] J.B. McLeod, On an infinite set of non-linear differential equations, Quart. J. Math. Oxford, 13, 119-128 (1962).

[25] R. Muralidar, D. Ramkrishna, An inverse problem in agglomeration kinetics, J. Colloid Interface Sci., 112, 348-361 (1986).

[26] V. Safronov, Evolution of the Protoplanetary Cloud and Formation of the Earth and the Planets, Israel Program for Scientific Translations, Jerusalem, 1972.

[27] M. Shirvani, H. van Roessel, The mass-conserving solutions of Smoluchowski's coagulation equation: The general bilinear kernel, Z. Angew. Math. Phys., 43, 526-535 (1992).

[28] M. Slemrod, A note on the kinetic equations of coagulation, J. Integral Eq. \& Appl., 3, 167-173 (1991).

[29] D.J. Smit, M.J. Hounslow, W.R. Paterson, Aggregation and gelation-I. Analytical solutions for CST and batch operation, II. Mixing effects in continuous flow vessels, III. Numerical classification of kernels and case studies of aggregation and growth, Chem. Eng. Sci., 49, 1025-1035 (1994), 49, 3147-3167 (1994), 50 849-862 (1995).

[30] M. Smoluchowski, Versuch einer mathematischen Theorie der Koagulationskinetik kolloider Lösungen, Z. Phys. Chem., 92, 129-168 (1917).

[31] G. Stell, R.M. Ziff, Kinetics of polymer gelation, J. Chem. Phys., 73, 3492-3499 (1980).

[32] W.H. Stockmayer, Theory of molecular size distribution and gel formation in branched chain polymers, J. Chem. Phys., 11, 45-55 (1943).

[33] R.M. Ziff, E.M. Hendriks, M.H. Ernst, Critical properties for gelation: A kinetic approach, Phys. Rev. Lett., 49, 593-595 (1982). 